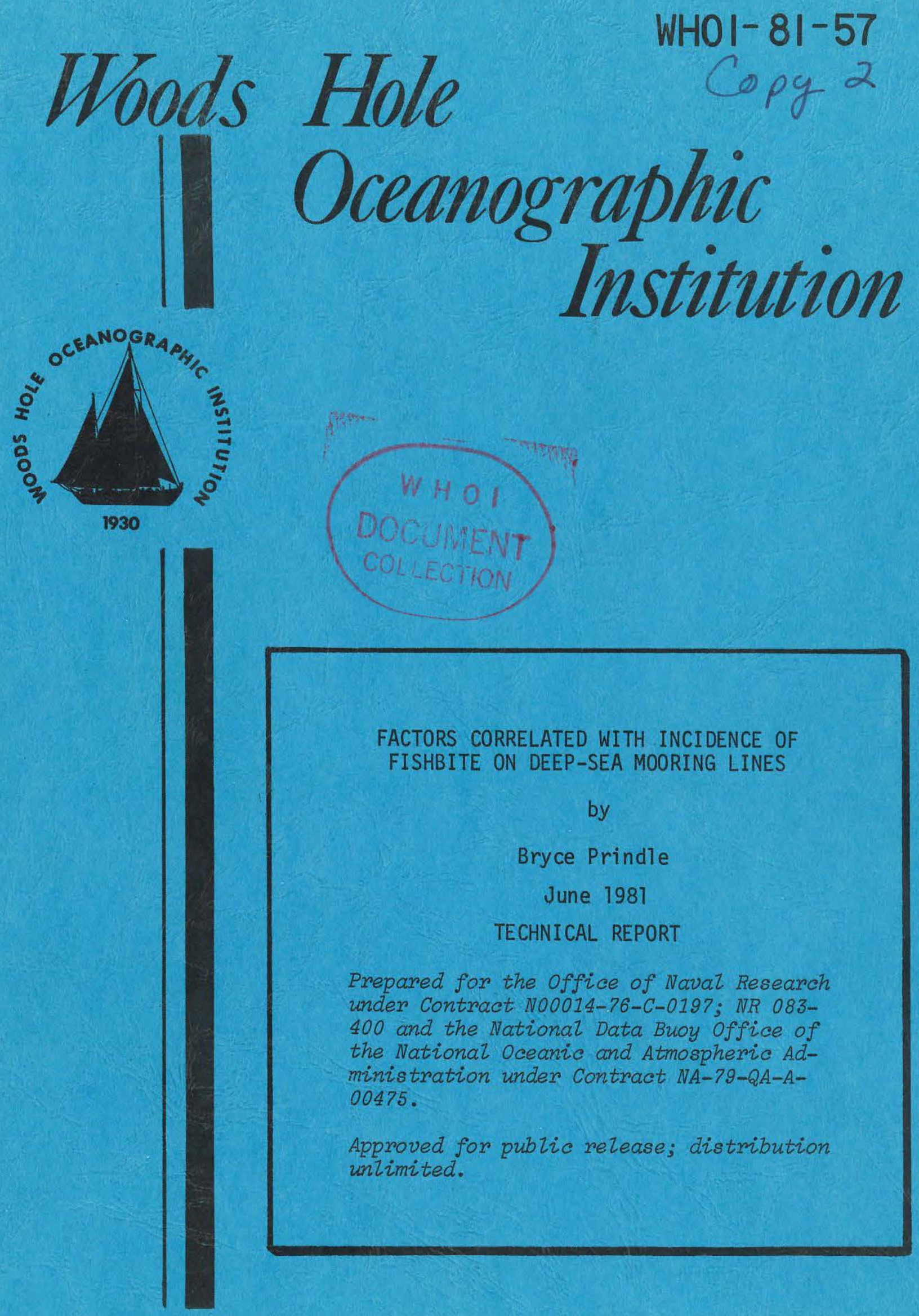

WOODS HOLE, MASSACHUSETTS 02543 
WHOI $-81-57$

\title{
FACTORS CORRELATED WITH INCIDENCE OF \\ FISHBITE ON DEEP-SEA MOORING LINES
}

\author{
by
}

Bryce Prindle

WOODS HOLE OCEANOGRAPHIC INSTITUTION

Woods Hole, Massachusetts 02543

June 1981

TECHNICAL REPORT

Prepared for the Office of Naval Research under Contract No0024-76-C-0297; NR 083-400 and the National Data Buoy Office of the National Oceanic and Atomospheric Administration under Contract NA-79-QA-A-00475.

Reproduction in whole or in part is permitted for any purpose of the United States Govermment. In citing this report in a bibliography, the reference given should be to: Woods Hole Oceanog. Inst. Tech. Rept. WHOI-81- 57.

Approved for public release; distribution unlimited.

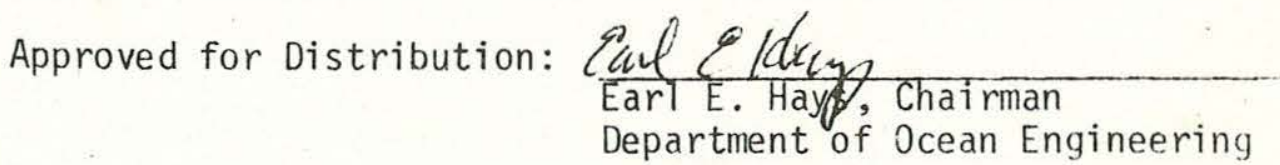


Abstract $\quad$ ii

Introduction 1

Procedures 1

Annual Variations 5

Fishbite vs Float Depth 7

Fishbite vs Latitude 9

Fishbite vs Depth of Water 9

Fishbite vs Duration 9

$\begin{array}{ll}\text { Conclusions } & 15\end{array}$

Appendix $: 16$

Tables VIII through XIX - Data from Woods Hole Oceanographic Institution Moored Stations

Bibliography

Figure 1 Fishbite at Moored Stations of the Woods Hole Pg. 5a Oceanographic Institution

$\begin{array}{lll}\text { Figure } 2 & \text { Fishbite vs Float Depth Pg. 8a }\end{array}$

Figure 3 Fishbite vs Latitude Pg. 10a 


\section{ACKNOWLEDGEMENTS}

The author wishes to express his appreciation of the efforts of Robert G. Walden in the inception and encouragement of the investigative work herein reported; and to the agencies which have provided financial support. The developments described in this report have been made possible by funds provided by the National Data Buoy Office of the National Oceanic and Atmospheric Administration under Contract NA-79-QA-A-00475 and by the Office of Naval Research under Contract N00014-76-C-0197. 


\begin{abstract}
Data from 399 moored stations established over the years 1967 through 1978 have been analyzed with reference to fishbite. Fishbite appears to have been a significant hazard as it was found in $22 \%$ of the lines placed within 40 degrees of the equator and where parts of the lines were at 2000 meters depth or less. Latitude, depth of water, and distance of mooring components beneath the surface were found to be correlated with incidence of fishbite; duration of a mooring was not.
\end{abstract}




\section{Introduction}

The occurrence and nature of fishbite damage to lines used in the deep-sea have been documented (Prindle and Walden, 1976), but questions have remained concerning the actual degree of risk which is to be expected as lines are placed in the ocean under various circumstances. Given a number of moored stations, what percentage of the mooring lines might one expect to be bitten? What are the relationships between fishbite and such factors as geographical location, depth of water, surface vs subsurface mooring floats, and the service life of a mooring?

In an attempt to find quantitative answers to such questions, data from 399 moored stations placed by the Woods Hole Oceanographic Institution from 1967 through 1978 were assembled and analyzed, correlating incidence of fishbite with -

1. Depth of mooring components

2. Mooring site

3. Depth of water at mooring sites

4. Duration of moored stations

\section{Procedure}

Log sheets of Woods Hole Oceanographic Institution's moored stations were reviewed and data relative to fishbite tabulated for the years 1967 through 1978. In a11, records of 399 stations are included in the study. 1967 was chosen as the starting year because it was the first year when fishbite observations were made on a routine basis. 1978 was the last year for which station logs were complete. The dates given for moorings are the dates of deployment. Moorings set each year are grouped together. As duration of moorings has increased, a number, of course, have not been recovered until sometime in the calendar year following that in which they were established. Nevertheless all are listed according to year of deployment. 
Only data which are thought to have a direct bearing on fishbite are included; mooring site, float depth, depth of water, duration, and of courge recorded observations of the occurrence or non-occurrence of fishbite. Except for moored station locations and fishbite records, data are used exactly as given in the station logs.

With reference to mooring site, two sets of figures for latitude and longitude are given in most logs reflecting either drifting or some variation in measurement of position at times of deployment and recovery. For purposes of this report, station location has been taken as the average between the two sets of data points.

Fishbite data have been reduced to "+", line bitten or "0", line not bitten, regardless of the number of bites found on any individual mooring line. It has been assumed that all lines were examined for evidence of fishbite and that in each case where typical damage was found a record of fishbite was made. In the cases of all other station logs, whether the record indicated a search for fishbite with negative results, or where a $\log$ contained no reference to fishbite, it has been assumed that the line was not bitten. Such a method may not lead to working figures which contain a record of every contact between lines and fish teeth. However, it would seem to be in line with practical considerations which govern the use of obviously damaged lines.

The fishbite data were recorded by personnel who happened to be aboard ship at the time of hauling. Hence, many observers with varied experience in detecting fishbite and often under pressure of other duties were involved. In the writer's experience, observers working under shipboard conditions usually do not find as many bites as a later, detailed examination of a line in the laboratory will revea1. The number of fishbites reported in the $\log$ sheets is therefore regarded as conservative. 
Data from Woods Hole Oceanographic Institution's moored stations deployed from 1967 through 1978 inclusive are tabulated in the Appendix to this report. Records of moored stations 256 through 654, a total of 399 stations, are included. Ocean Areas Included in the Study

385 , or $96 \%$, of all moored stations in the study were deployed in the north Atlantic Ocean. The remaining 14 were placed: five in the Pacific Ocean near Hawai, four in the Phillipine Sea, and five in the Indian Ocean. In terms of world ocean space, therefore, the representation of data is predominately from the Atlantic Ocean north of the equator. What follows by way of interpretation of the data can be applied to that area with some degree of confidence. With reference to other parts of the world ocean, however, conclusions can only be tentative until more uniform coverage has been obtained.

of the total number of stations, 261 or $65 \%$ were located in what will hereinafter be designated as the "fishbite zone". It is an ocean space bounded by latitude and by depth. It lies between $40^{\circ}$ north and $40^{\circ}$ south latitude. The depth boundaries are between the water-air interface and 2000 meters below the surface. The boundaries are based upon experience with deep-sea moorings and upon other information in the "Deep-Sea Lines Fishbite Manual, (Prindle and Walden, 1976). In the time period covered in the present report, 19 Woods Hole Oceanographic Institution buoys were deployed outside the area bounded by the $40^{\circ}$ north and south parallels. None of those lines were bitten. Data for the same are presented in Table I. However, 11 of the 19 had a11 components below 2000 meters depth, so only eight were inside what has already been designated as the space where biting is likely to occur. The present data support the 
$-4-$

Table I

WHOI Stations Moored Above $40^{\circ}$ Latitude

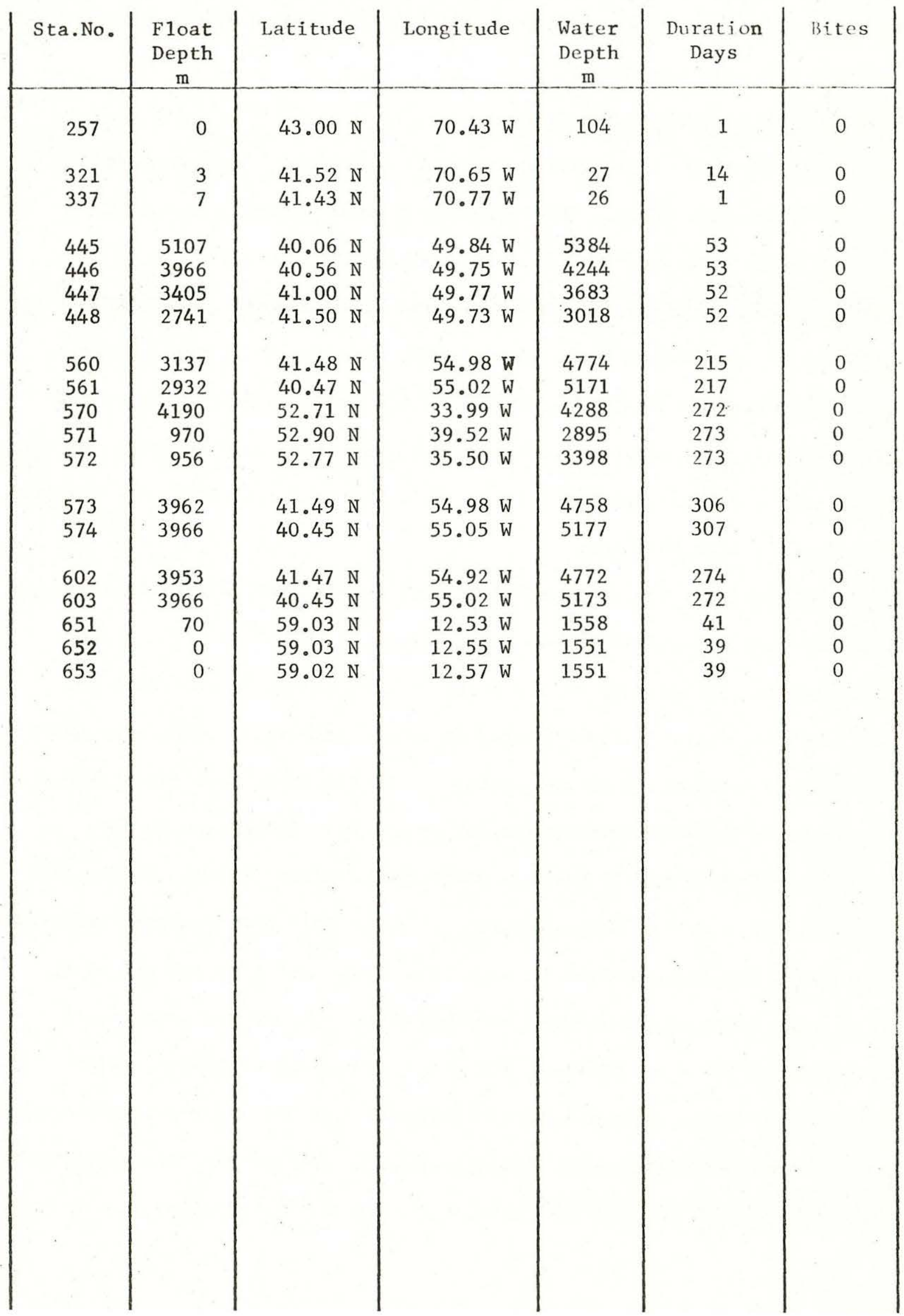


use of $40^{\circ}$ latitude as a boundary for the fishbite zone, but more information would be desirable.

With reference to depth, 96 moored arrays were placed inside the $40^{\circ}$ parallels but with float depths greater than 2000 meters. Of these, none were reported bitten.

For purposes of this report, it will be assumed that moored station components located outside $40^{\circ}$ north or south latitude and at depths greater than 2000 meters have been exposed to negligible risk of fishbite and will be considered to have been outside the fishbite zone. For a number of purposes, the incidence of biting will be calculated upon the basis of number of deployments within the delineated zone.

261 , or $65 \%$, of the total number of successfully completed and documented moorings were within the fishbite zone. Biting appears to have been a significant hazard as $22 \%$ of the mooring lines from within that group were reported to have developed markings characteristic of fishbite. Data for the same are summarized in Table II。 Seasona1 Variations

Fishbite attack appears to have been quite variable from one year to another as is illustrated in Figure 1. For example, in 1968 no lines were reported to have been bitten; the next year, at the same location, (Site $\mathrm{D}, 39^{\circ} \mathrm{N}$ Lat., $70^{\circ} \mathrm{W}$ Long.) with a like number of lines exposed, the attack rate was 27\%. From 1975 through 1978, the rate of attack at all stations appears to have been on the increase, rising from $8 \%$ to $62 \%$ of lines placed within the fishbite zone. Interesting, if true. Taking the data as they stand in the record, several possibilities appear. One is that fishbite hazard may vary from time to time at the same location, especially if it is near the boundary of the fishbite zone. Site D is such a location. In 1968, 21 stations with buoys above 


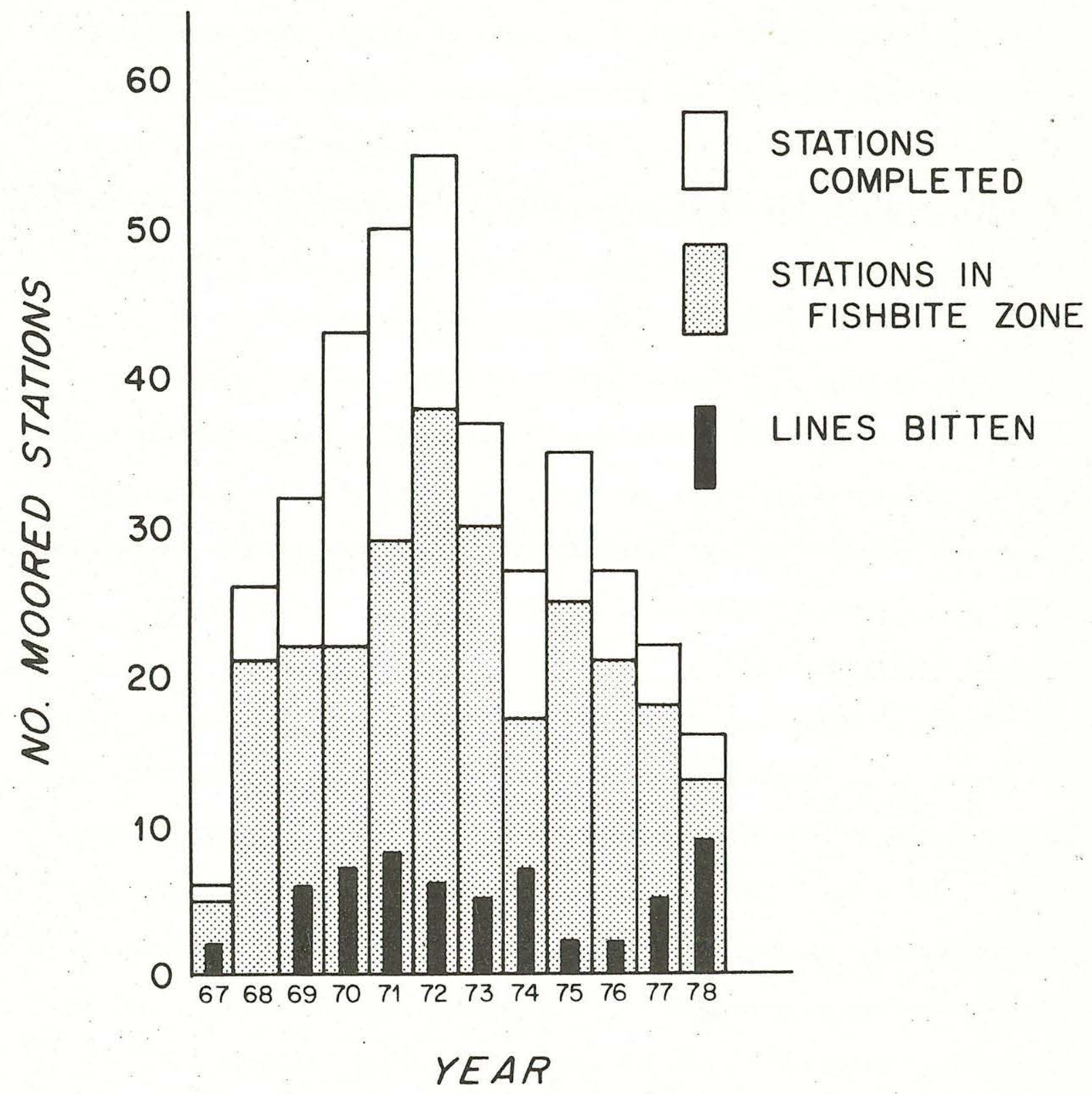

FIGURE 1: Fishbite at WH0I Moored Stations \#256-\#654 
Table II

Incidence of Fishbite

on

WHOI Moored Stations in the Fishbite Zone

\begin{tabular}{|c|c|c|c|}
\hline $\begin{array}{l}\text { Year } \\
\text { Set }\end{array}$ & $\begin{array}{l}\text { Stations } \\
\text { Completed }\end{array}$ & $\begin{array}{l}\text { Lines } \\
\text { No. }\end{array}$ & $\begin{array}{c}\text { Bitten } \\
\%\end{array}$ \\
\hline 1967 & 5 & 2 & 40 \\
\hline 1968 & 21 & 0 & 0 \\
\hline 1969 & 22 & 6 & 27 \\
\hline 1970 & 22 & 7 & 32 \\
\hline 1971 & 29 & 8 & 28 \\
\hline 1972 & 38 & 6 & 16 \\
\hline 1973 & 30 & 5 & 17 \\
\hline 1974 & 17 & 7 & 41 \\
\hline 1975 & 25 & 2 & 8 \\
\hline 1976 & 21 & 2 & 10 \\
\hline 1977 & 18 & 5 & 28 \\
\hline 1978 & 13 & 8 & 62 \\
\hline Overa11 & 261 & 58 & 22 \\
\hline
\end{tabular}


2000 meters depth were completed at Site D, and the record indicates that none of them were bitten. Indeed, 19 of the mooring lines were unprotected synthetic fiber and only one array was lost. The rest were all on station and appeared unbitten after durations of up to 180 days. In 1969, 22 buoys were moored in the same manner at Site D. Six of them, or $27 \%$, had bitten lines when they were recovered. The data suggest that there had been some change at Site $D$, and in fact it is possible that a meandering of the Gulf Stream, put the edge of it over Site D in 1969 and that with the Stream came warm water, sharks, and perhaps other biting organisms.

The apparent rise in incidence of fishbite between the years of 1975 and 1977 is more difficult to explain. There does not seem to be a clear correlation between the trend and any recorded conditions of exposure. Data samples were small and decreased from 1975 to 1978. Possibly the increase may have been due to more acute observations of recovered lines as there were fewer to study.

\section{Fishbite vs Float Depth}

Previous experience, as reviewed in the Deep-Sea Lines Fishbite Manual, seemed to indicate that mooring lines with surface floats were more susceptible to fishbite than were those with subsurface floats. Results of analysis of the present data, assembled in Table III, however, seem to indicate that fishbite risk is about $30 \%$ at a11 depths to 500 meters. Beyond the 500 meter leve1, incidence of fishbite fell off until beyond a depth of 1500 meters there was no record of fishbite having occurred. Despite this record, however, it should be noted that data from other sources have indicated the occurrence of fishbite at depths ranging to 2000 meters. The 2000 meter depth should continue to be regarded as the minimum depth for use of non-bite-resistant lines in the fishbite zone. 
Table III

WHOI Moored Stations 256 Through 654

A11 stations between $40^{\circ} \mathrm{N} \& \mathrm{~S}$ Latitude

\begin{tabular}{|c|c|c|c|}
\hline $\begin{array}{l}\text { Float } \\
\text { Depth } \\
\text { Meters }\end{array}$ & $\begin{array}{l}\text { Total } \\
\text { Number }\end{array}$ & $\begin{array}{l}\text { Number } \\
\text { Bitten }\end{array}$ & $\begin{array}{c}\% \\
\text { Bitten }\end{array}$ \\
\hline 0 & 90 & 27 & 30 \\
\hline 1- 99 & 6 & 0 & \\
\hline $100-199$ & 28 & 8 & 29 \\
\hline $200-299$ & 11 & 2 & \\
\hline $300-399$ & 3 & 0 & \\
\hline $400-499$ & 43 & 16 & 37 \\
\hline $500-599$ & 31 & 3 & 10 \\
\hline $600-699$ & 3 & 1 & \\
\hline $700-799$ & 1 & 0 & \\
\hline $800-899$ & 2 & 0 & \\
\hline $900-999$ & 11 & 0. & \\
\hline $1000-1099$ & 5 & 0 & \\
\hline $1100-1199$ & 0 & 0 & \\
\hline $1200-1299$ & 1 & 0 & \\
\hline $1300-1399$ & 1 & 0 & \\
\hline $1400-1499$ & 9 & 1 & \\
\hline $1500-1599$ & 1 & 0 & \\
\hline $1600-1699$ & 2 & 0 & \\
\hline $1700-1799$ & 1 & 0 & \\
\hline $1800-1899$ & 0 & 0 & \\
\hline $1900-1999$ & 11 & 0 & \\
\hline $2000+$ & 103 & 0 & 0 \\
\hline
\end{tabular}


$-8 a-$

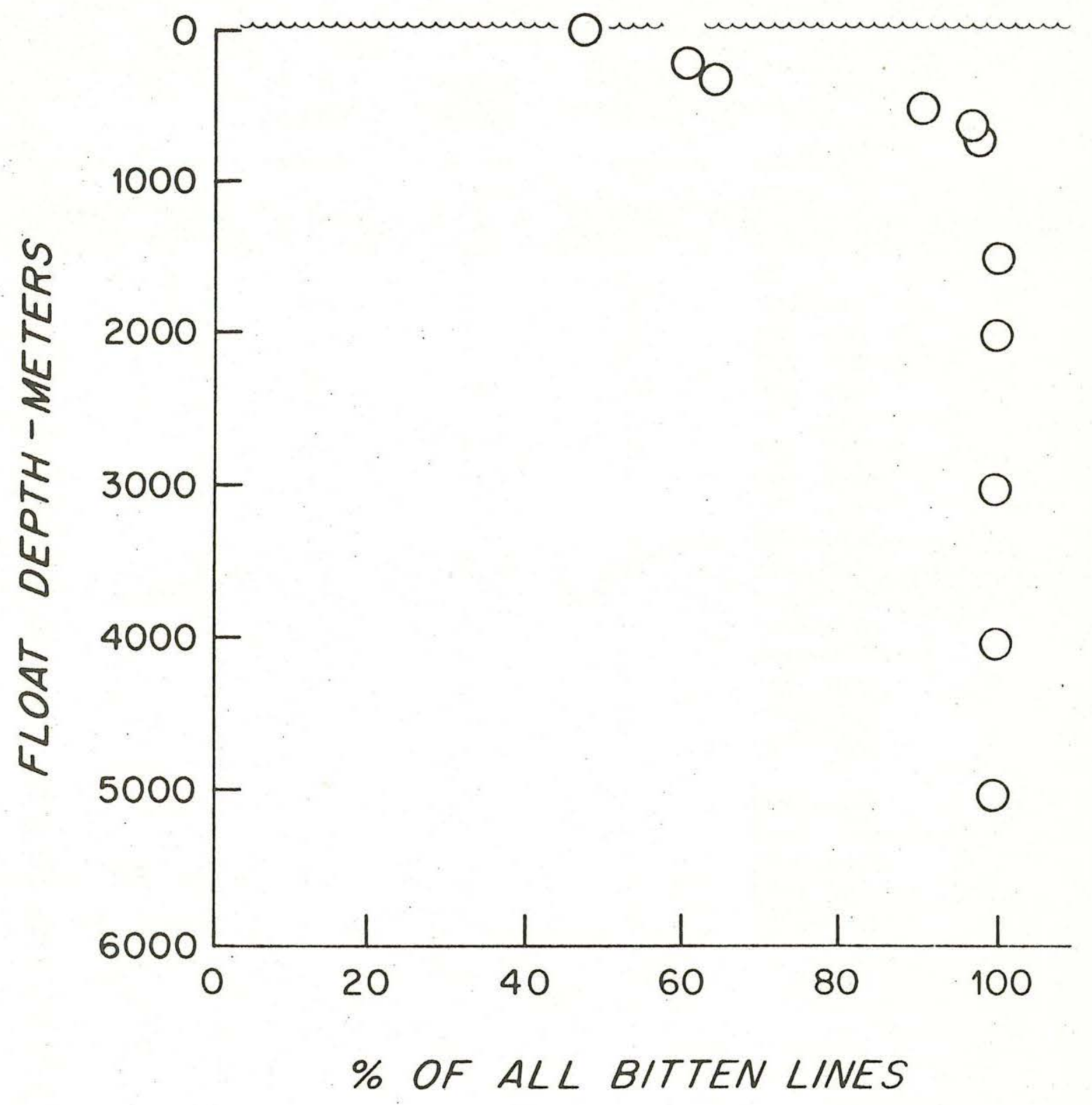

FIGURE 2: Fishbite vs Float Depth WHOI Moored Stations \#256-\#654 


\section{Fishbite vs Latitude}

One may well ask whether risk of fishbite was found to be uniform throughout the fishbite zone as bounded by the $40^{\circ}$ parallels. The data indicate that it was not. The risk rose as stations were established closer to the equator. Considering the data in Table IV, it appears that in the three ranges of latitude where significant numbers of mooring were established, there was a close relationship between the percentage of lines bitten and the latitude at which a moored station was located. Using the three points noted, one can derive an equation: $y=-0.427 x+44.29$ where " $\mathrm{x}$ " is the \% of all lines in the fishbite zone bitten within a five degree range of latitude and "y" is the average latitude of all lines within the same five degree range of latitude. The derived equation fits the observed facts we11. As illustrated in Figure 3, the three data points lie very close to a line drawn using the equation. Furthermore, the equation predicts that there will be no lines bitten above $44^{\circ}$ latitude; and $100 \%$ of the lines will be bitten when they are placed within $1.59^{\circ}$ of the equator. The latter is what happened to 11 lines recently exposed at latitudes between $0.04^{\circ} \mathrm{S}$ and $0.782 \mathrm{~N}$ in the Indian Ocean. Fishbite vs Depth of Water

To date, fishbite has been regarded as mostly a deep water phenomenon. Insofar as they go, the present data confirm such a viewpoint. No fishbites were recorded at 26 stations in 2000 meters of water or less, though all were within latitudes where fishbite had been encountered in deeper water. Until more evidence becomes available, however, one should probably not write off the possibility that fishbite may occur in shallow water. There is a wide range of conditions in water less than 2000 meters deep.

\section{Fishbite vs Duration}

One might surmise that the time a mooring line is in the water should 
Table IV

\section{Fishbite vs Latitude}

WHOI Moored Stations 256 Through 654

Al1 lines wholly or partially at 0 to $2000 \mathrm{~m}$ depth

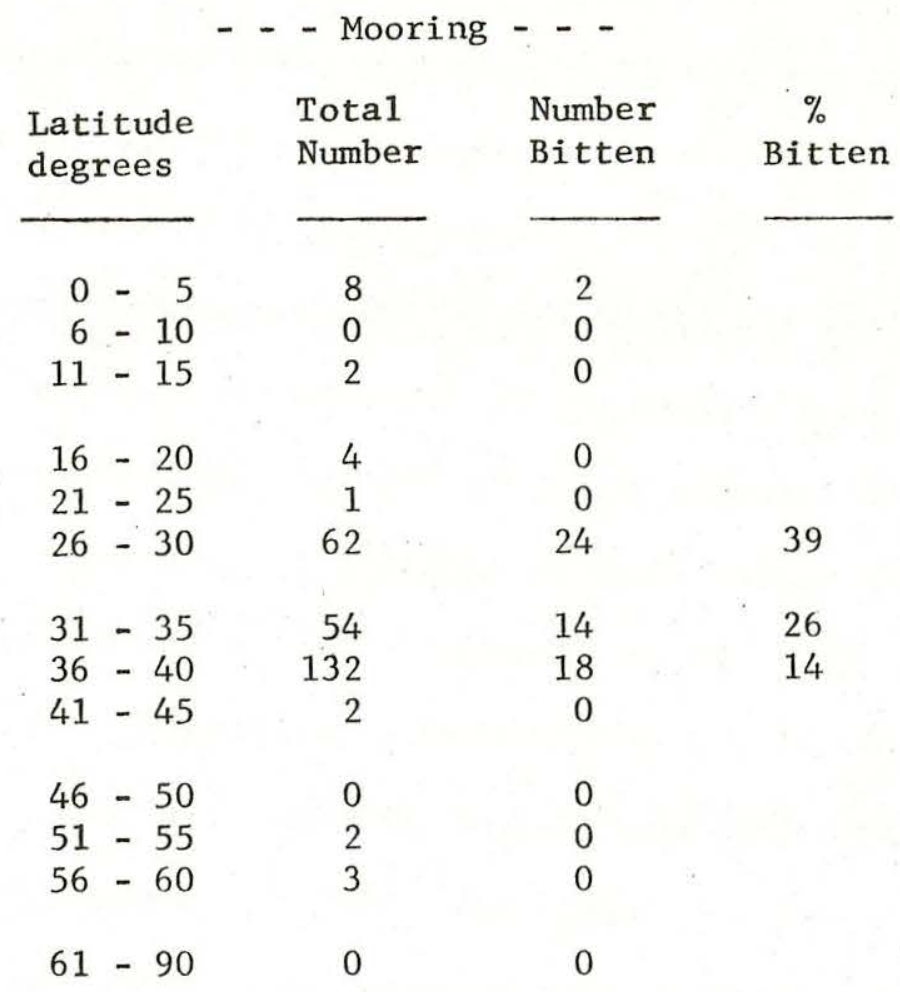




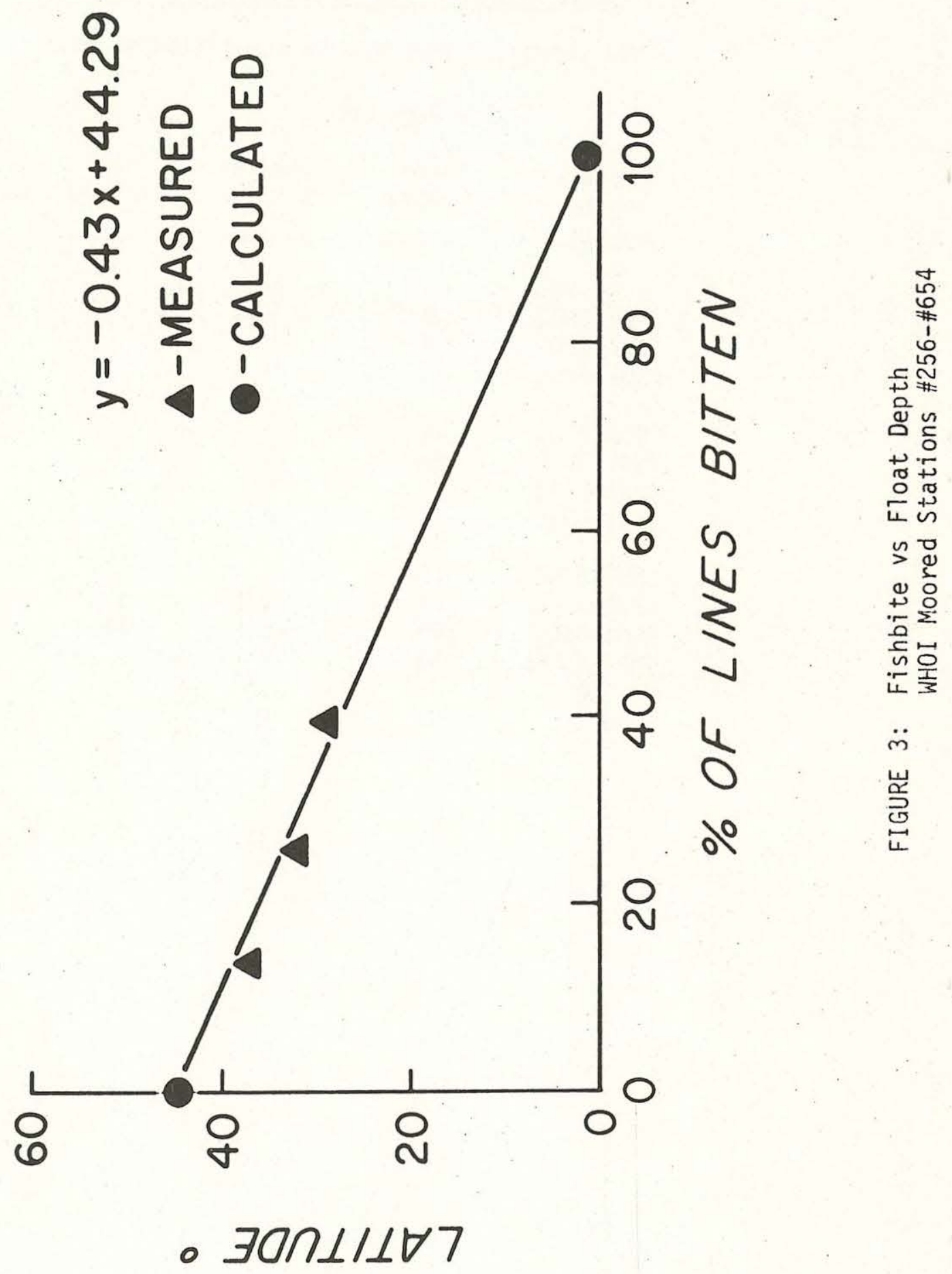


Table V

Fishbite vs Depth of Water

WHOI Moored Stations 256 Through 654

A11 mooring lines within the fishbite zone

\begin{tabular}{|c|c|c|c|}
\hline $\begin{array}{l}\text { Water } \\
\text { Depth } \\
\text { meters }\end{array}$ & $\begin{array}{l}\text { Tota1 } \\
\text { Number }\end{array}$ & $\begin{array}{l}\text { Number } \\
\text { Bitten }\end{array}$ & $\begin{array}{c}\% \\
\text { Bitten }\end{array}$ \\
\hline $0-500$ & 4 & 0 & \\
\hline $501-1000$ & 9 & 0 & \\
\hline $1001-1500$ & 6 & 0 & \\
\hline $1501-2000$ & 7 & 0 & \\
\hline $2001-2500$ & 4 & 3 & \\
\hline $2501-3000$ & 86 & 11 & 13 \\
\hline $3001-3500$ & 1 & 0 & \\
\hline $3501-4000$ & 7 & 5 & \\
\hline $4001-4500$ & 13 & 3 & \\
\hline $4501-5000$ & 13 & 3 & \\
\hline $5001-5500$ & 100 & 29 & 29 \\
\hline $5501-6000$ & 10 & 4 & \\
\hline $6000+$ & 1 & 1 & \\
\hline
\end{tabular}


have some correlation with the probability that it will be bitten. Does longer duration increase risk of fishbite? Is there a minimum time for bites to develop; or is biting a random occurrence over a period of time?

The record of bites vs duration is given in Table VI. An attempt to analyze the data by the method of linear regression indicates that there was no correlation between the times that lines were in the water and the percentage of lines bitten. This would indicate that biting is a random occurrence vs time; that within the time span studied, up to 515 days, only certain lines, $22 \%$ of the total, became susceptible to biting; the majority did not. Another possibility is that lines were bitten mainly during deployment or recovery, not while on station.

Looking at the data another way, in which the duration times of stations hauled after a short time are considered to be samples of longer times at sea, and the data are summarized, as in Table VII, so that all bites up to a certain time are grouped together, it appears that the percentage of 1 ines bitten increased from $13 \%$ in 10 days or 1 ess to $22 \%$ in 450 days. Results of this type of analysis suggest that the percentage of lines bitten may indeed increase with time but may approach a limit; in this case, $22 \%$ at 450 days duration. Why $22 \%$ should be a saturation point, if indeed it is, is not understood at present. More work is needed to establish a clear relationship between the time a mooring line is in the water and the occurrence of fishbite. 
Table VI

\section{Fishbite vs Duration}

WHOI Moored Stations 256 Through 654

\begin{tabular}{|c|c|c|c|}
\hline $\begin{array}{l}\text { Duration } \\
\text { Days }\end{array}$ & $\begin{array}{l}\text { Total } \\
\text { Number }\end{array}$ & $\begin{array}{l}\text { Number } \\
\text { Bitten }\end{array}$ & $\begin{array}{c}\% \\
\text { Bitten }\end{array}$ \\
\hline $0-10$ & 38 & 5 & 13 \\
\hline $11-50$ & 31 & 4 & 13 \\
\hline $51-100$ & 47 & 11 & 23 \\
\hline $101-150$ & 45 & 5 & 11 \\
\hline $151-200$ & 18 & 10 & \\
\hline $201-250$ & 29 & 4 & 14 \\
\hline $251-300$ & 19 & 6 & \\
\hline $301-350$ & 15 & 4 & \\
\hline $351-400$ & 8 & 2 & \\
\hline $401-450$ & 9 & 5 & \\
\hline $451-500$ & 1 & 0 & \\
\hline $501-550$ & 2 & 2 & \\
\hline $551+$ & 0 & & \\
\hline
\end{tabular}


Table VII

Fishbite vs Duration

WHOI Moored Stations 256 Through 654

A11 mooring lines within the fishbite zone

\begin{tabular}{|c|c|c|c|}
\hline $\begin{array}{l}\text { Duration } \\
\text { up to } \\
\text { - days } \\
\end{array}$ & $\begin{array}{l}\text { Total } \\
\text { Number }\end{array}$ & $\begin{array}{l}\text { Number } \\
\text { Bitten }\end{array}$ & $\begin{array}{c}\% \\
\text { Bitten }\end{array}$ \\
\hline-10 & 38 & 5 & 13 \\
\hline-50 & 69 & 9 & 13 \\
\hline-100 & 116 & 20 & 17 \\
\hline-150 & 161 & 25 & 16 \\
\hline-200 & 179 & 35 & 20 \\
\hline-250 & 208 & 39 & 19 \\
\hline-300 & 227 & 45 & 20 \\
\hline-350 & 242 & 49 & 20 \\
\hline-400 & 250 & 51 & 20 \\
\hline-450 & 259 & 56 & 22 \\
\hline-500 & 260 & 56 & 22 \\
\hline-550 & 262 & 58 & 22 \\
\hline
\end{tabular}




\section{$\underline{\text { Conclusions }}$}

Analysis of data from 399 Woods Hole Oceanographic Institution moored stations, numbered 256 through 654 and established in the years 1967 through 1978, leads to the following conclusions:

1. Fishbite was found to have occurred only within an ocean space designated as the fishbite zone which was bounded by $40^{\circ}$ north and south parallels and depth levels of 0 and 2000 meters.

2. Fishbite is a significant hazard to deep-sea mooring lines. It was reported on $22 \%$ of al1 lines from moorings within the fishbite zone.

3. Risk of fishbite was found to increase linearly with latitude from $0 \%$ of exposed lines at approximately $44^{\circ}$ north latitude to $100 \%$ within $1.6^{\circ}$ of the equator.

4. Within the fishbite zone, mooring lines had a uniform rate of fishbite risk throughout the top 500 meters of water. Below: 500 meters, fishbite hazard fell off and was close to zero at 2000 meters depth.

5. A correlation between fishbite and the overall water depth at a mooring site is indicated. No fishbite occurred at 26 stations within the fishbite zone where water was shallow, i.e. 1ess than 2001 meters deep.

6. Incidence of fishbite had no clear correlation with the length of time a mooring line was in the water. This may indicate that lines are bitten mainly during deployment and/or recovery。 
$-16-$

APPENDIX 
Table VIII

WHOI Moored Station Data Year - 1967

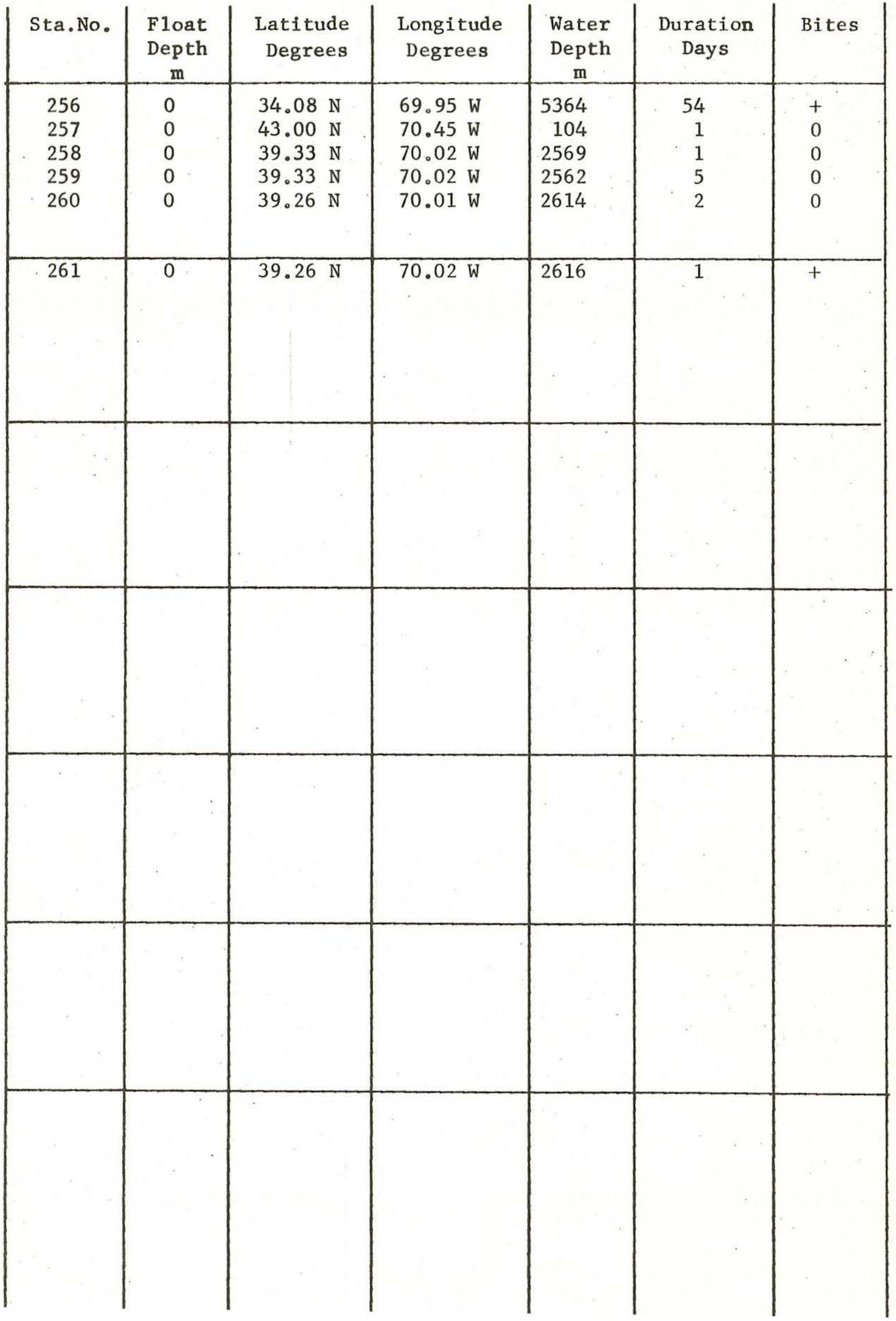


Table IX

WHOI Moored Station Data Year - 1968

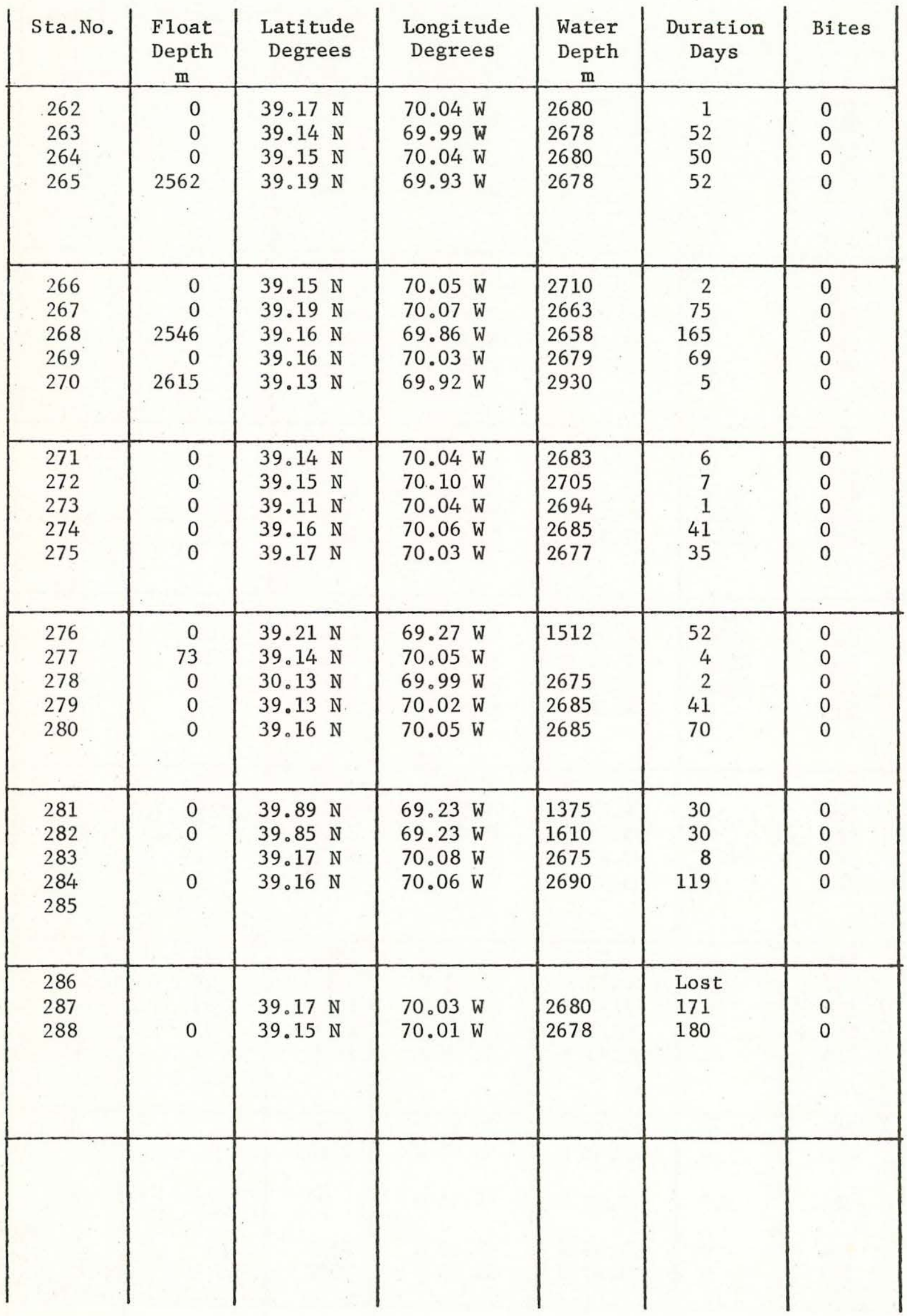


Table X

WHOI Moored Station Data Year - 1969

\begin{tabular}{|c|c|c|c|c|c|c|}
\hline Sta.No. & $\begin{array}{c}\text { Float } \\
\text { Depth } \\
\mathrm{m}\end{array}$ & $\begin{array}{r}\text { Latitude } \\
\text { Degrees }\end{array}$ & $\begin{array}{l}\text { Longitude } \\
\text { Degrees }\end{array}$ & $\begin{array}{c}\text { Water } \\
\text { Depth } \\
\mathrm{m}\end{array}$ & $\begin{array}{c}\text { Duration } \\
\text { Days }\end{array}$ & Bites \\
\hline $\begin{array}{l}289 \\
290 \\
291 \\
292 \\
293\end{array}$ & $\begin{array}{r}0 \\
0 \\
0 \\
2563\end{array}$ & $\begin{array}{l}38.02 \mathrm{~N} \\
39.19 \mathrm{~N} \\
39.15 \mathrm{~N} \\
39.14 \mathrm{~N} \\
39.16 \mathrm{~N}\end{array}$ & $\begin{array}{r}4.99 \mathrm{E} \\
70.02 \mathrm{~W} \\
70.04 \mathrm{~W} \\
69.91 \mathrm{~W} \\
70.05 \mathrm{~W}\end{array}$ & $\begin{array}{l}2833 \\
2682 \\
2682 \\
2686 \\
2678\end{array}$ & $\begin{array}{r}49 \\
8 \\
8 \\
2 \\
7\end{array}$ & $\begin{array}{l}0 \\
0 \\
0 \\
0 \\
0\end{array}$ \\
\hline $\begin{array}{l}294 \\
295 \\
296 \\
297 \\
298\end{array}$ & $\begin{array}{l}0 \\
0 \\
0\end{array}$ & $\begin{array}{l}39.17 \mathrm{~N} \\
39.17 \mathrm{~N} \\
39.18 \mathrm{~N} \\
39.18 \mathrm{~N} \\
39.15 \mathrm{~N}\end{array}$ & $\begin{array}{l}70.00 \mathrm{~W} \\
70.07 \mathrm{~W} \\
70.03 \mathrm{~W} \\
70.03 \mathrm{~W} \\
69.98 \mathrm{~W}\end{array}$ & $\begin{array}{l}2674 \\
2690 \\
2674 \\
2672 \\
2675\end{array}$ & $\begin{array}{l}9 \\
3 \\
2 \\
1\end{array}$ & $\begin{array}{l}0 \\
0 \\
0 \\
0 \\
+\end{array}$ \\
\hline $\begin{array}{l}299 \\
300 \\
301 \\
302 \\
303\end{array}$ & $\begin{array}{r}0 \\
0 \\
0 \\
2577 \\
2624\end{array}$ & $\begin{array}{l}39.19 \mathrm{~N} \\
39.16 \mathrm{~N} \\
39.16 \mathrm{~N} \\
39.11 \mathrm{~N} \\
39.11 \mathrm{~N}\end{array}$ & $\begin{array}{l}70.65 \mathrm{~W} \\
70.03 \mathrm{~W} \\
69.94 \mathrm{~W} \\
70.00 \mathrm{~W} \\
70.04 \mathrm{~W}\end{array}$ & $\begin{array}{l}2680 \\
2680 \\
2685 \\
2692\end{array}$ & $\begin{array}{r}16 \\
105 \\
4 \\
126 \\
1\end{array}$ & $\begin{array}{l}0 \\
+ \\
0 \\
0 \\
0\end{array}$ \\
\hline $\begin{array}{l}304 \\
305 \\
306 \\
307 \\
308\end{array}$ & $\begin{array}{r}4266 \\
4206 \\
4148 \\
4061 \\
0\end{array}$ & $\begin{array}{ll}36.42 & \mathrm{~N} \\
36.72 & \mathrm{~N} \\
37.03 & \mathrm{~N} \\
37.34 \mathrm{~N} & 39.16 \mathrm{~N}\end{array}$ & $\begin{array}{l}70.00 \mathrm{~W} \\
70.00 \mathrm{~W} \\
70.00 \mathrm{~W} \\
70.02 \mathrm{~W} \\
69.90 \mathrm{~W}\end{array}$ & $\begin{array}{l}4486 \\
4426 \\
4368 \\
4281 \\
2682\end{array}$ & $\begin{array}{r}69 \\
69 \\
69 \\
69 \\
120\end{array}$ & $\begin{array}{l}0 \\
0 \\
0 \\
0 \\
0\end{array}$ \\
\hline $\begin{array}{l}309 \\
310 \\
311 \\
312 \\
313\end{array}$ & $\begin{array}{r}0 \\
0 \\
3974 \\
0\end{array}$ & $\begin{array}{l}39.15 \mathrm{~N} \\
39.16 \mathrm{~N} \\
39.19 \mathrm{~N} \\
37.93 \mathrm{~N} \\
33.99 \mathrm{~N}\end{array}$ & $\begin{array}{l}70.00 \mathrm{~W} \\
70.05 \mathrm{~W} \\
70.08 \mathrm{~W} \\
70.00 \mathrm{~W} \\
70.04 \mathrm{~W}\end{array}$ & $\begin{array}{l}2678 \\
2683 \\
2685 \\
4088 \\
5368\end{array}$ & $\begin{array}{r}59 \\
146 \\
56 \\
1 \\
2\end{array}$ & $\begin{array}{l}+ \\
+ \\
0 \\
0 \\
0\end{array}$ \\
\hline $\begin{array}{l}314 \\
315 \\
316 \\
317 \\
318\end{array}$ & $\begin{array}{l}0 \\
0 \\
0 \\
0 \\
0\end{array}$ & $\begin{array}{l}34.03 \mathrm{~N} \\
34.02 \mathrm{~N} \\
39.11 \mathrm{~N} \\
39.19 \mathrm{~N} \\
39.33 \mathrm{~N}\end{array}$ & $\begin{array}{l}70.02 \mathrm{~W} \\
69.97 \mathrm{~W} \\
70.01 \mathrm{~W} \\
70.08 \mathrm{~W} \\
70.04 \mathrm{~W}\end{array}$ & $\begin{array}{l}5368 \\
5368 \\
2692 \\
2681 \\
2545\end{array}$ & $\begin{array}{l}51 \\
51 \\
92 \\
91 \\
92\end{array}$ & $\begin{array}{l}+ \\
0 \\
+ \\
+ \\
+\end{array}$ \\
\hline $\begin{array}{l}319 \\
320\end{array}$ & $\begin{array}{l}0 \\
0\end{array}$ & $\begin{array}{l}33.98 \mathrm{~N} \\
34.02 \mathrm{~N}\end{array}$ & $\begin{array}{l}70.01 \mathrm{~W} \\
70.07 \mathrm{~W}\end{array}$ & $\begin{array}{l}5370 \\
5370\end{array}$ & 2 & 0 \\
\hline 321 & 2.7 & $41.52 \mathrm{~N}$ & $70.65 \mathrm{~W}$ & 27 & 114 & 0 \\
\hline $\begin{array}{l}324 \\
325\end{array}$ & $\begin{array}{l}0 \\
0\end{array}$ & $\begin{array}{l}31.83 \mathrm{~N} \\
31.83 \mathrm{~N}\end{array}$ & $\begin{array}{l}65.25 \mathrm{~W} \\
65.25 \mathrm{~W}\end{array}$ & $\begin{array}{l}2646 \\
2921\end{array}$ & & \\
\hline
\end{tabular}


Table XI

WHOI Moored Station Data Year -1970

\begin{tabular}{|c|c|c|c|c|c|c|}
\hline Sta.No. & $\begin{array}{c}\text { Float } \\
\text { Depth } \\
\mathrm{m}\end{array}$ & $\begin{array}{r}\text { Latitude } \\
\text { Degrees }\end{array}$ & $\begin{array}{l}\text { Longitude } \\
\text { Degrees }\end{array}$ & $\begin{array}{c}\text { Water } \\
\text { Depth } \\
\text { m }\end{array}$ & $\begin{array}{l}\text { Duration } \\
\text { Days }\end{array}$ & Bites \\
\hline $\begin{array}{l}322 \\
323 \\
324 \\
325\end{array}$ & $\begin{array}{l}0 \\
0\end{array}$ & $\begin{array}{l}38.64 \mathrm{~N} \\
33.98 \mathrm{~N}\end{array}$ & $\begin{array}{l}69.32 \mathrm{~W} \\
69.97 \mathrm{~W}\end{array}$ & $\begin{array}{l}2690 \\
5365\end{array}$ & $\begin{array}{r}55 \\
125\end{array}$ & $\begin{array}{l}0 \\
0\end{array}$ \\
\hline $\begin{array}{l}326 \\
327 \\
328 \\
329 \\
330\end{array}$ & $\begin{array}{l}3988 \\
4203 \\
4243 \\
4236 \\
5464\end{array}$ & $\begin{array}{ll}37.64 & \mathrm{~N} \\
36.77 & \mathrm{~N} \\
31.00 \mathrm{~N} \\
31.00 \mathrm{~N} \\
27.99 \mathrm{~N}\end{array}$ & $\begin{array}{l}70.01 \mathrm{~W} \\
70.00 \mathrm{~W} \\
69.30 \mathrm{~W} \\
70.49 \mathrm{~W} \\
69.97 \mathrm{~W}\end{array}$ & $\begin{array}{l}4128 \\
4417 \\
5356 \\
5424 \\
5464\end{array}$ & $\begin{array}{l}130 \\
130 \\
124 \\
124 \\
122\end{array}$ & $\begin{array}{l}0 \\
0 \\
0 \\
0 \\
0\end{array}$ \\
\hline $\begin{array}{l}331 \\
332 \\
333 \\
334 \\
335\end{array}$ & $\begin{array}{r}219 \\
417 \\
4 \\
0 \\
1300\end{array}$ & $\begin{array}{l}11.54 \mathrm{~N} \\
11.65 \mathrm{~N} \\
32.08 \mathrm{~N} \\
33.97 \mathrm{~N} \\
32.13 \mathrm{~N}\end{array}$ & $\begin{array}{l}61.90 \mathrm{~W} \\
61.90 \mathrm{~W} \\
64.19 \mathrm{~W} \\
69.93 \mathrm{~W} \\
64.13 \mathrm{~W}\end{array}$ & $\begin{array}{r}477 \\
675 \\
4384 \\
5370 \\
4400\end{array}$ & $\begin{array}{l}37 \\
37 \\
48 \\
83 \\
46\end{array}$ & $\begin{array}{l}0 \\
0 \\
0 \\
0 \\
0\end{array}$ \\
\hline $\begin{array}{l}336 \\
337 \\
338 \\
339 \\
340\end{array}$ & $\begin{array}{r}5284 \\
7 \\
0 \\
0 \\
0\end{array}$ & $\begin{array}{l}33.98 \mathrm{~N} \\
41.43 \mathrm{~N} \\
39.58 \mathrm{~N} \\
39.13 \mathrm{~N} \\
39.12 \mathrm{~N}\end{array}$ & $\begin{array}{l}69.94 \mathrm{~W} \\
70.77 \mathrm{~W} \\
69.91 \mathrm{~W} \\
70.03 \mathrm{~W} \\
70.64 \mathrm{~W}\end{array}$ & $\begin{array}{r}5370 \\
26 \\
2322 \\
2682 \\
2754\end{array}$ & $\begin{array}{r}238 \\
1 \\
51 \\
50 \\
51\end{array}$ & $\begin{array}{l}0 \\
0 \\
+ \\
+ \\
0\end{array}$ \\
\hline $\begin{array}{l}341 \\
342 \\
343 \\
344 \\
345\end{array}$ & $\begin{array}{r}0 \\
0 \\
2253 \\
0 \\
1492\end{array}$ & $\begin{array}{l}34.04 \mathrm{~N} \\
33.95 \mathrm{~N} \\
35.98 \mathrm{~N} \\
33.98 \mathrm{~N} \\
39.39 \mathrm{~N}\end{array}$ & $\begin{array}{l}70.00 \mathrm{~W} \\
69.81 \mathrm{~W} \\
70.55 \mathrm{~W} \\
69.98 \mathrm{~W} \\
70.98 \mathrm{~W}\end{array}$ & $\begin{array}{l}5365 \\
5363 \\
4444 \\
5365 \\
2527\end{array}$ & $\begin{array}{r}45 \\
6 \\
56 \\
56 \\
49\end{array}$ & $\begin{array}{l}+ \\
0 \\
0 \\
+ \\
0\end{array}$ \\
\hline $\begin{array}{l}346 \\
347 \\
348 \\
349 \\
350\end{array}$ & $\begin{array}{r}2155 \\
766 \\
964 \\
843 \\
885\end{array}$ & $\begin{array}{l}39.60 \mathrm{~N} \\
39.82 \mathrm{~N} \\
39.84 \mathrm{~N} \\
39.84 \mathrm{~N} \\
39.83 \mathrm{~N}\end{array}$ & $\begin{array}{l}70.96 \mathrm{~W} \\
70.65 \mathrm{~W} \\
70.95 \mathrm{~W} \\
70.94 \mathrm{~W} \\
70.93 \mathrm{~W}\end{array}$ & $\begin{array}{r}2263 \\
876 \\
977 \\
943 \\
993\end{array}$ & $\begin{array}{r}115 \\
105 \\
48 \\
48 \\
107\end{array}$ & $\begin{array}{l}0 \\
0 \\
0 \\
0 \\
0\end{array}$ \\
\hline $\begin{array}{l}351 \\
352 \\
353 \\
354 \\
355\end{array}$ & $\begin{array}{r}2049 \\
2377 \\
4108 \\
5281 \\
0\end{array}$ & $\begin{array}{ll}39.61 & \mathrm{~N} \\
39.39 & \mathrm{~N} \\
35.97 & \mathrm{~N} \\
34.03 & \mathrm{~N} \\
33.97 & \mathrm{~N}\end{array}$ & $\begin{array}{l}71.24 \mathrm{~W} \\
71.03 \mathrm{~W} \\
70.57 \mathrm{~W} \\
69.98 \mathrm{~W} \\
69.93 \mathrm{~W}\end{array}$ & $\begin{array}{l}2150 \\
2509 \\
4436 \\
5368 \\
5361\end{array}$ & $\begin{array}{r}114 \\
66 \\
62 \\
207 \\
60\end{array}$ & $\begin{array}{l}0 \\
0 \\
0 \\
0 \\
+\end{array}$ \\
\hline $\begin{array}{l}356 \\
357 \\
358 \\
359 \\
360\end{array}$ & $\begin{array}{r}0 \\
2044 \\
1454 \\
3310 \\
3668\end{array}$ & $\begin{array}{ll}33.80 & \mathrm{~N} \\
35.97 & \mathrm{~N} \\
39.13 & \mathrm{~N} \\
37.28 & \mathrm{~N} \\
36.39 & \mathrm{~N}\end{array}$ & $\begin{array}{l}70.20 \mathrm{~W} \\
70.92 \mathrm{~W} \\
70.02 \mathrm{~W} \\
71.82 \mathrm{~W} \\
71.25 \mathrm{~W}\end{array}$ & $\begin{array}{l}5374 \\
4425 \\
2680 \\
3528 \\
4230\end{array}$ & $\begin{array}{l}\text { Lost } \\
148 \\
137 \\
139 \\
141\end{array}$ & $\begin{array}{l}0 \\
0 \\
0 \\
0\end{array}$ \\
\hline & & & & & & \\
\hline
\end{tabular}


Table XI - cont'd.

WHOI Moored Station Data Year - 1970

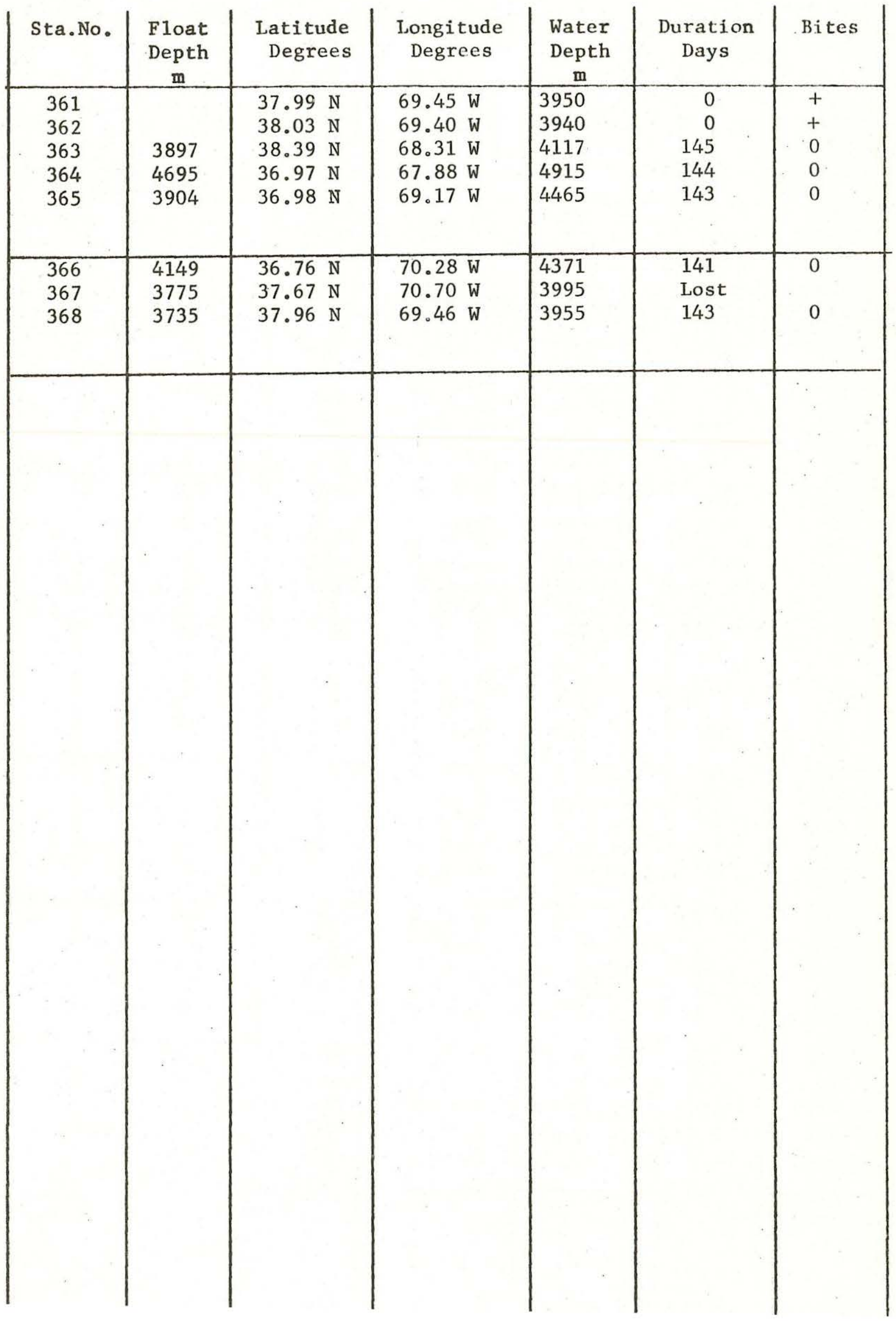


Table XII

WHOI Moored Station Data Year - 1971

\begin{tabular}{|c|c|c|c|c|c|c|}
\hline Sta.No. & $\begin{array}{c}\text { Float } \\
\text { Depth } \\
\text { m }\end{array}$ & $\begin{array}{r}\text { Latitude } \\
\text { Degrees }\end{array}$ & $\begin{array}{l}\text { Longitude } \\
\text { Degrees }\end{array}$ & $\begin{array}{c}\text { Water } \\
\text { Depth } \\
\text { m }\end{array}$ & $\begin{array}{c}\text { Duration } \\
\text { Days }\end{array}$ & Bites \\
\hline $\begin{array}{l}369 \\
370\end{array}$ & $\begin{array}{l}5601 \\
5186\end{array}$ & $\begin{array}{l}22.80 \mathrm{~N} \\
22.25 \mathrm{~N}\end{array}$ & $\begin{array}{l}66.48 \mathrm{~W} \\
67.32 \mathrm{~W}\end{array}$ & $\begin{array}{l}5817 \\
5402\end{array}$ & $\begin{array}{l}122 \\
121\end{array}$ & $\begin{array}{l}0 \\
0\end{array}$ \\
\hline $\begin{array}{l}371 \\
372 \\
373 \\
374 \\
375\end{array}$ & $\begin{array}{r}5294 \\
132 \\
0 \\
0 \\
0\end{array}$ & $\begin{array}{rl}21.27 & \mathrm{~N} \\
0.37 & \mathrm{~N} \\
1.00 \mathrm{~N} \\
0.00 \mathrm{~N} \\
1.04 \mathrm{~N}\end{array}$ & $\begin{array}{r}68.00 \mathrm{~W} \\
160.03 \mathrm{~W} \\
150.00 \mathrm{~W} \\
149.92 \mathrm{~W} \\
149.85 \mathrm{~W}\end{array}$ & $\begin{array}{r}5326 \\
183 \\
4441 \\
4451 \\
4647\end{array}$ & $\begin{array}{c}118 \\
\text { Lost } \\
223 \\
7 \\
2\end{array}$ & $\begin{array}{l}0 \\
0 \\
+\end{array}$ \\
\hline $\begin{array}{l}376 \\
377 \\
378 \\
379 \\
380\end{array}$ & $\begin{array}{l}0 \\
0 \\
0\end{array}$ & $\begin{array}{r}1.00 \mathrm{~N} \\
39.13 \mathrm{~N} \\
39.13 \mathrm{~N} \\
39.14 \mathrm{~N} \\
37.34 \mathrm{~N}\end{array}$ & $\begin{array}{r}150.00 \mathrm{~W} \\
70.00 \mathrm{~W} \\
69.99 \mathrm{~W} \\
69.99 \mathrm{~W} \\
70.37 \mathrm{~W}\end{array}$ & $\begin{array}{l}4431 \\
2665 \\
2665 \\
2662 \\
4160\end{array}$ & $\begin{array}{c}\text { Lost } \\
27 \\
27 \\
91 \\
2\end{array}$ & $\begin{array}{l}0 \\
0 \\
0 \\
+\end{array}$ \\
\hline $\begin{array}{l}381 \\
382 \\
383 \\
384 \\
385\end{array}$ & $\begin{array}{r}0 \\
2059 \\
4516 \\
3395 \\
1031\end{array}$ & $\begin{array}{l}33.96 \mathrm{~N} \\
35.97 \mathrm{~N} \\
39.84 \mathrm{~N} \\
33.01 \mathrm{~N} \\
32.80 \mathrm{~N}\end{array}$ & $\begin{array}{r}69.96 \mathrm{~W} \\
7.0 .50 \mathrm{~W} \\
48.50 \mathrm{~W} \\
136.58 \mathrm{E} \\
134.71 \mathrm{E}\end{array}$ & $\begin{array}{l}5375 \\
4445 \\
4803 \\
3578 \\
1211\end{array}$ & $\begin{array}{r}184 \\
87 \\
91 \\
108 \\
106\end{array}$ & $\begin{array}{l}+ \\
0 \\
0 \\
0 \\
0\end{array}$ \\
\hline $\begin{array}{l}386 \\
387 \\
388 \\
389 \\
390\end{array}$ & $\begin{array}{r}880 \\
2058 \\
4775 \\
4766 \\
4770\end{array}$ & $\begin{array}{ll}32.97 & \mathrm{~N} \\
31.52 & \mathrm{~N} \\
37.76 & \mathrm{~N} \\
37.95 & \mathrm{~N} \\
38.17 & \mathrm{~N}\end{array}$ & $\begin{array}{r}134.30 \mathrm{E} \\
132.48 \mathrm{E} \\
64.49 \mathrm{~W} \\
64.66 \mathrm{~W} \\
64.80 \mathrm{~W}\end{array}$ & $\begin{array}{r}880 \\
2236 \\
5005 \\
4996 \\
5000\end{array}$ & $\begin{array}{c}\text { Lost } \\
128 \\
32 \\
32 \\
31\end{array}$ & $\begin{array}{l}0 \\
0 \\
0 \\
0\end{array}$ \\
\hline $\begin{array}{l}391 \\
392 \\
393 \\
394 \\
395\end{array}$ & $\begin{array}{r}4701 \\
4640 \\
4580 \\
4550 \\
0\end{array}$ & $\begin{array}{l}38.39 \mathrm{~N} \\
38.58 \mathrm{~N} \\
38.80 \mathrm{~N} \\
39.00 \mathrm{~N} \\
39.54 \mathrm{~N}\end{array}$ & $\begin{array}{l}64.97 \mathrm{~W} \\
65.16 \mathrm{~W} \\
65.37 \mathrm{~W} \\
65.52 \mathrm{~W} \\
69.96 \mathrm{~W}\end{array}$ & $\begin{array}{l}4931 \\
4870 \\
4810 \\
4780 \\
2428\end{array}$ & $\begin{array}{l}31 \\
31 \\
31 \\
30 \\
45\end{array}$ & $\begin{array}{l}0 \\
0 \\
0 \\
0 \\
+\end{array}$ \\
\hline $\begin{array}{l}396 \\
397 \\
398 \\
399 \\
400\end{array}$ & $\begin{array}{r}0 \\
0 \\
0 \\
0 \\
2023\end{array}$ & $\begin{array}{l}39.16 \mathrm{~N} \\
39.19 \mathrm{~N} \\
39.13 \mathrm{~N} \\
39.20 \mathrm{~N} \\
35.97 \mathrm{~N}\end{array}$ & $\begin{array}{l}70.13 \mathrm{~W} \\
69.93 \mathrm{~W} \\
70.00 \mathrm{~W} \\
69.26 \mathrm{~W} \\
70.44 \mathrm{~W}\end{array}$ & $\begin{array}{l}2738 \\
2655 \\
2660 \\
2927 \\
4447\end{array}$ & $\begin{array}{r}102 \\
106 \\
88 \\
8 \\
136\end{array}$ & $\begin{array}{l}0 \\
+ \\
0 \\
0 \\
0\end{array}$ \\
\hline $\begin{array}{l}401 \\
402 \\
403 \\
404 \\
405\end{array}$ & $\begin{array}{r}0 \\
0 \\
0 \\
5265 \\
0\end{array}$ & $\begin{array}{ll}33.87 & \mathrm{~N} \\
39.00 \mathrm{~N} \\
35.93 \mathrm{~N} \\
34.01 \mathrm{~N} \\
33.99 \mathrm{~N}\end{array}$ & $\begin{array}{l}69.99 \mathrm{~W} \\
70.01 \mathrm{~W} \\
70.30 \mathrm{~W} \\
70.02 \mathrm{~W} \\
70.10 \mathrm{~W}\end{array}$ & $\begin{array}{l}5363 \\
2754 \\
4465 \\
5368 \\
5373\end{array}$ & $\begin{array}{r}85 \\
93 \\
20 \\
377 \\
109\end{array}$ & $\begin{array}{l}0 \\
0 \\
0 \\
0 \\
0\end{array}$ \\
\hline $\begin{array}{l}406 \\
407 \\
408 \\
409 \\
410\end{array}$ & $\begin{array}{r}0 \\
0 \\
1483 \\
1502 \\
1484\end{array}$ & $\begin{array}{ll}28.00 & N \\
28.01 & N \\
27.81 & N \\
28.03 & N \\
28.36 & N\end{array}$ & $\begin{array}{l}70.00 \mathrm{~W} \\
70.35 \mathrm{~W} \\
70.15 \mathrm{~W} \\
70.11 \mathrm{~W} \\
69.69 \mathrm{~W}\end{array}$ & $\begin{array}{l}5460 \\
5465 \\
5470 \\
5465 \\
5460\end{array}$ & $\begin{array}{l}101 \\
102 \\
102 \\
102 \\
101\end{array}$ & $\begin{array}{l}+ \\
+ \\
0 \\
0 \\
0\end{array}$ \\
\hline
\end{tabular}


Table XII - cont'd。

WHOI Moored Station Data Year - 1971

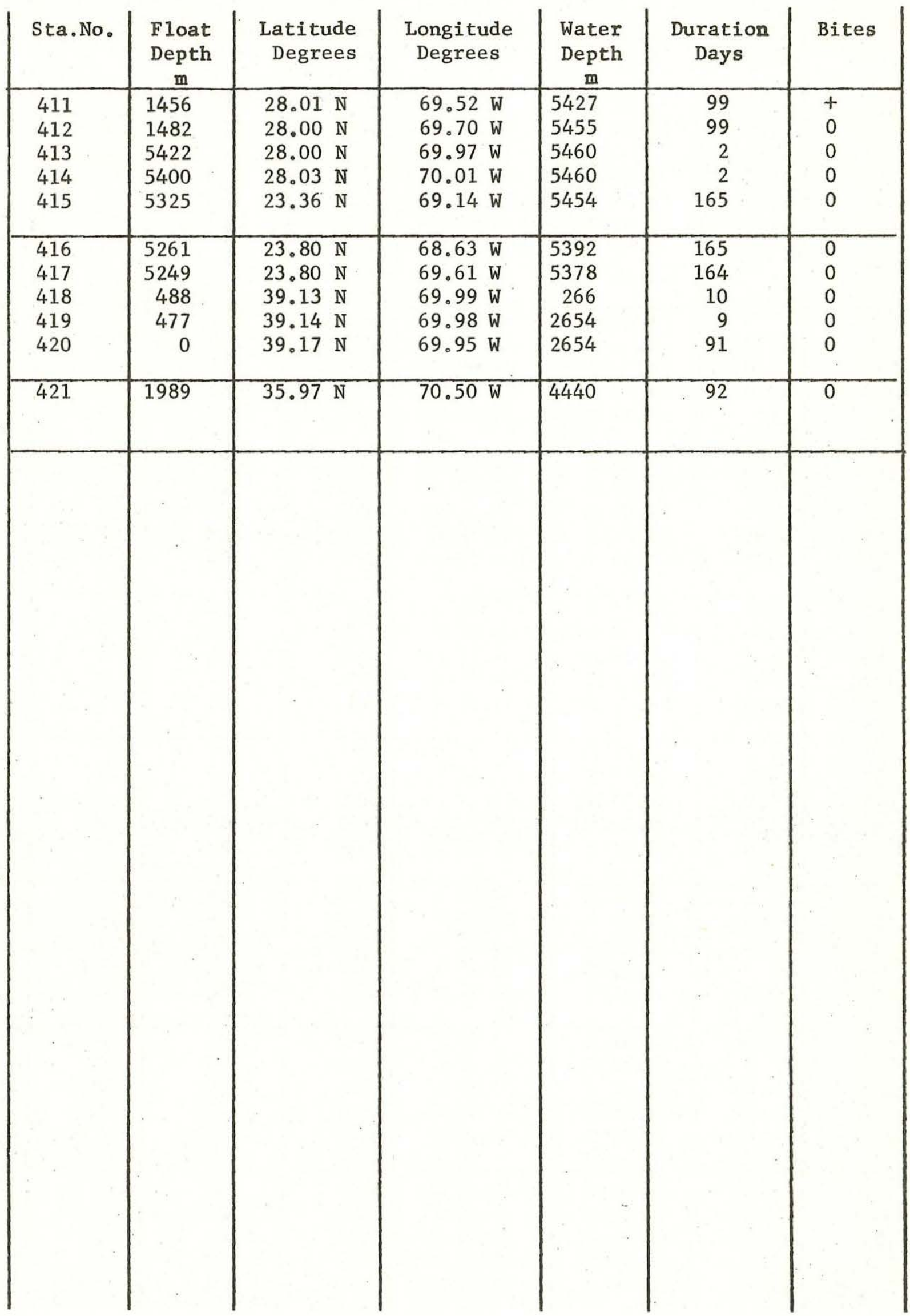


Table XIII

WHOI Moored Station Data Year - 1972

\begin{tabular}{|c|c|c|c|c|c|c|}
\hline Sta.No. & $\begin{array}{c}\text { Float } \\
\text { Depth } \\
\mathrm{m}\end{array}$ & $\begin{array}{r}\text { Latitude } \\
\text { Degrees }\end{array}$ & $\begin{array}{l}\text { Longitude } \\
\text { Degrees }\end{array}$ & $\begin{array}{c}\text { Water } \\
\text { Depth } \\
\text { m }\end{array}$ & $\begin{array}{l}\text { Duration } \\
\text { Days }\end{array}$ & Bites \\
\hline 422 & 1009 & $39.04 \mathrm{~N}$ & $70.04 \mathrm{~W}$ & 2724 & 117 & 0 \\
\hline 423 & 999 & $39.18 \mathrm{~N}$ & $70.56 \mathrm{~W}$ & 2729 & 107 & 0 \\
\hline 424 & 0 & $28.17 \mathrm{~N}$ & $68.62 \mathrm{~W}$ & 5254 & 111 & 0 \\
\hline 425 & 0 & $28.00 \mathrm{~N}$ & $69.66 \mathrm{~W}$ & 5462 & Lost & \\
\hline 426 & 1690 & $17.61 \mathrm{~N}$ & $65.25 \mathrm{~W}$ & 1756 & 39 & 0 \\
\hline 427 & 1727 & $23.60 \mathrm{~N}$ & $65.24 \mathrm{~W}$ & 1809 & 39 & 0 \\
\hline 428 & 0 & $39.21 \mathrm{~N}$ & $65.97 \mathrm{~W}$ & 2640 & 1 & 0 \\
\hline 429 & 0 & $39.17 \mathrm{~N}$ & $69.99 \mathrm{~W}$ & 2656 & 172 & + \\
\hline 430 & 3962 & $28.16 \mathrm{~N}$ & $68.57 \mathrm{~W}$ & 5221 & 68 & 0 \\
\hline $\begin{array}{l}431 \\
432\end{array}$ & 1440 & $28.34 \mathrm{~N}$ & $68.40 \mathrm{~W}$ & 5370 & $\begin{array}{c}68 \\
\text { Lost }\end{array}$ & 0 \\
\hline 433 & 1470 & $28.18 \mathrm{~N}$ & $68.39 \mathrm{~W}$ & 5380 & 68 & 0 \\
\hline 434 & 1450 & $28.16 \mathrm{~N}$ & $68.20 \mathrm{~W}$ & 5275 & 67 & 0 \\
\hline 435 & 437 & $27.98 \mathrm{~N}$ & $68.41 \mathrm{~W}$ & 5280 & 66 & 0 \\
\hline 436 & 150 & $39.85 \mathrm{~N}$ & $70.09 \mathrm{~W}$ & 1072 & 71 & 0 \\
\hline 437 & 5200 & $37.00 \mathrm{~N}$ & $49.74 \mathrm{~W}$ & 5477 & 59 & 0 \\
\hline 438 & 5144 & $37.51 \mathrm{~N}$ & $49.74 \mathrm{~W}$ & 5421 & 58 & 0 \\
\hline 439 & 5150 & $38.16 \mathrm{~N}$ & $49.77 \mathrm{~W}$ & 5412 & 58 & 0 \\
\hline 440 & 5142 & $38.29 \mathrm{~N}$ & $49.78 \mathrm{~W}$ & 5419 & 56 & 0 \\
\hline 441 & 4562 & $38.65 \mathrm{~N}$ & $49.79 \mathrm{~W}$ & 5419 & 56 & 0 \\
\hline 442 & 4559 & $39.00 \mathrm{~N}$ & $49.77 \mathrm{~W}$ & 5416 & 56 & 0 \\
\hline 443 & 4559 & $39.39 \mathrm{~N}$ & $49.77 \mathrm{~W}$ & 5416 & 55 & 0 \\
\hline 444 & 4556 & $39.67 \mathrm{~N}$ & $49.70 \mathrm{~W}$ & 5413 & 54 & 0 \\
\hline 445 & 5107 & $40.06 \mathrm{~N}$ & $49.84 \mathrm{~W}$ & 5384 & 53 & 0 \\
\hline 446 & 3966 & $40.56 \mathrm{~N}$ & $49.75 \mathrm{~W}$ & 4244 & 53 & 0 \\
\hline 447 & 3405 & $41.00 \mathrm{~N}$ & $49.77 \mathrm{~W}$ & 3683 & 52 & 0 \\
\hline 448 & 2741 & $41.50 \mathrm{~N}$ & $49.73 \mathrm{~W}$ & 3018 & 52 & 0 \\
\hline 449 & 1030 & $39.97 \mathrm{~N}$ & $69.98 \mathrm{~W}$ & 2769 & 102 & 0 \\
\hline 450 & 995 & $39.15 \mathrm{~N}$ & $70.51 \mathrm{~W}$ & 2754 & 102 & 0 \\
\hline 451 & 0 & $28.90 \mathrm{~N}$ & $69.69 \mathrm{~W}$ & 5437 & 162 & + \\
\hline 452 & 540 & $27.99 \mathrm{~N}$ & $70.65 \mathrm{~W}$ & 5452 & 161 & + \\
\hline 453 & 0 & $28.16 \mathrm{~N}$ & $68.63 \mathrm{~W}$ & 5261 & 158 & + \\
\hline 454 & 0 & $27.56 \mathrm{~N}$ & $69.69 \mathrm{~W}$ & 5462 & 156 & + \\
\hline 455 & 0 & $28.02 \mathrm{~N}$ & $69.63 \mathrm{~W}$ & 5462 & 160 & \\
\hline 456 & 1999 & $33.68 \mathrm{~N}$ & $62.59 \mathrm{~W}$ & 2998 & 147 & 0 \\
\hline 457 & 2894 & $33.68 \mathrm{~N}$ & $62.87 \mathrm{~W}$ & 4817 & Lost & \\
\hline 458 & 1949 & $39.61 \mathrm{~N}$ & $70.00 \mathrm{~W}$ & 2263 & 147 & 0 \\
\hline 459 & 2594 & $39.17 \mathrm{~N}$ & $70.24 \mathrm{~W}$ & 2709 & 150 & 0 \\
\hline 460 & 2349 & $39.16 \mathrm{~N}$ & $70.07 \mathrm{~W}$ & 2664 & 150 & 0 \\
\hline 461 & 2554 & $39.06 \mathrm{~N}$ & $70.00 \mathrm{~W}$ & 2669 & Lost & \\
\hline 462 & 23 & $39.91 \mathrm{~N}$ & $70.84 \mathrm{~W}$ & 501 & 21 & 0 \\
\hline 463 & 0 & $39.22 \mathrm{~N}$ & $70.05 \mathrm{~W}$ & 2646 & 72 & 0 \\
\hline 464 & 107 & $39.21 \mathrm{~N}$ & $70.05 \mathrm{~W}$ & 2649 & 11 & 0 \\
\hline 1.65 & ahe & $38.98 \mathrm{~N}$ & $70.00 \mathrm{~W}$ & 2756 & 103 & 0 \\
\hline
\end{tabular}


$-25-$

Table XIII - cont'd.

WHOI Moored Station Data Year - 1972

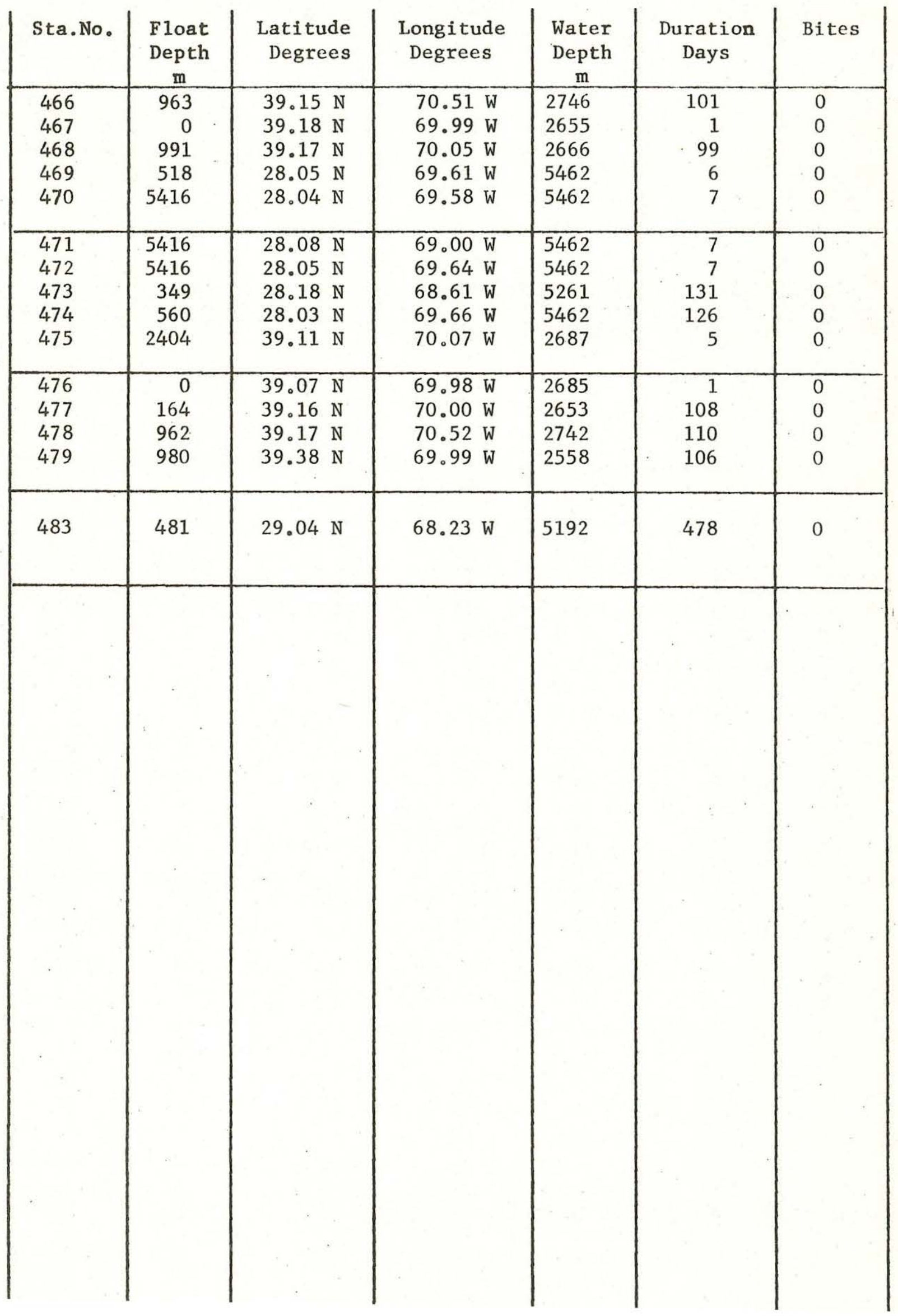


Table XIV

WHOI Moored Station Data Year - 1973

\begin{tabular}{|c|c|c|c|c|c|c|}
\hline Sta.No. & $\begin{array}{c}\text { Float } \\
\text { Depth } \\
\text { m }\end{array}$ & $\begin{array}{r}\text { Latitude } \\
\text { Degrees }\end{array}$ & $\begin{array}{c}\text { Longitude } \\
\text { Degrees }\end{array}$ & $\begin{array}{c}\text { Water } \\
\text { Depth } \\
\mathrm{m}\end{array}$ & $\begin{array}{c}\text { Duration } \\
\text { Days }\end{array}$ & Bites \\
\hline 480 & 0 & $28.06 \mathrm{~N}$ & $69.65 \mathrm{~W}$ & 5462 & Lost & \\
\hline 481 & 476 & $27.98 \mathrm{~N}$ & $69.67 \mathrm{~W}$ & 5462 & 116 & 0 \\
\hline 482 & 476 & $28.16 \mathrm{~N}$ & $68.65 \mathrm{~W}$ & 5239 & 106 & 0 \\
\hline 484 & 495 & $27.40 \mathrm{~N}$ & $67.98 \mathrm{~W}$ & 5151 & 112 & 0 \\
\hline 485 & 490 & $26.40 \mathrm{~N}$ & $69.34 \mathrm{~W}$ & 5420 & 111 & + \\
\hline 486 & 474 & $26.94 \mathrm{~N}$ & $71.08 \mathrm{~W}$ & 5474 & 110 & 0 \\
\hline 487 & 488 & $28.55 \mathrm{~N}$ & $71.38 \mathrm{~W}$ & 5327 & 1 & 0 \\
\hline 488 & 489 & $28.52 \mathrm{~N}$ & $71.39 \mathrm{~W}$ & 5325 & 108 & 0 \\
\hline 489 & 489 & $29.60 \mathrm{~N}$ & $69.99 \mathrm{~W}$ & 5440 & 106 & 0 \\
\hline 490 & 973 & $39.36 \mathrm{~N}$ & $69.99 \mathrm{~W}$ & 2559 & 203 & 0 \\
\hline 491 & 180 & $39.13 \mathrm{~N}$ & $69.97 \mathrm{~W}$ & 2654 & 204 & 0 \\
\hline 492 & 985 & $39.16 \mathrm{~N}$ & $70.51 \mathrm{~W}$ & 2749 & 201 & 0 \\
\hline 493 & 471 & $28.70 \mathrm{~N}$ & $70.27 \mathrm{~W}$ & 5446 & 91 & + \\
\hline 494 & 472 & $27.83 \mathrm{~N}$ & $70.66 \mathrm{~W}$ & 5446 & 89 & 0 \\
\hline 495 & 476 & $27.14 \mathrm{~N}$ & $70.01 \mathrm{~W}$ & 5477 & 89 & 0 \\
\hline 496 & 453 & & & 5286 & 1 & 0 \\
\hline 497 & 463 & $27.80 \mathrm{~N}$ & $69.02 \mathrm{~W}$ & 5296 & 87 & 0 \\
\hline 498 & 478 & $27.55 \mathrm{~N}$ & $69.57 \mathrm{~W}$ & 5463 & 86 & 0 \\
\hline 499 & 478 & $28.15 \mathrm{~N}$ & $70.14 \mathrm{~W}$ & 5461 & 87 & 0 \\
\hline 500 & 474 & $28.28 \mathrm{~N}$ & $69.28 \mathrm{~W}$ & 5456 & 84 & 0 \\
\hline 501 & 472 & $28.84 \mathrm{~N}$ & $69.31 \mathrm{~W}$ & 5379 & 87 & 0 \\
\hline 502 & 450 & $28.15 \mathrm{~N}$ & $68.69 \mathrm{~W}$ & 5255 & 231 & + \\
\hline 503 & 466 & $28.00 \mathrm{~N}$ & $69.74 \mathrm{~W}$ & 5461 & 170 & + \\
\hline 504 & & $20.31 \mathrm{~N}$ & $73.67 \mathrm{~W}$ & 1503 & & 0 \\
\hline 505 & & $20.28 \mathrm{~N}$ & $73.63 \mathrm{~W}$ & 1505 & & 0 \\
\hline 506 & 168 & $39.38 \mathrm{~N}$ & $70.00 \mathrm{~W}$ & 2559 & 237 & 0 \\
\hline 507 & 178 & $39.16 \mathrm{~N}$ & $70.01 \mathrm{~W}$ & 2662 & 176 & 0 \\
\hline 508 & 2613 & $39.17 \mathrm{~N}$ & $70.18 \mathrm{~W}$ & 2714 & 52 & 0 \\
\hline 509 & 160 & $39.14 \mathrm{~N}$ & $70.55 \mathrm{~W}$ & 2746 & 176 & 0 \\
\hline 510 & 0 & $27.74 \mathrm{~N}$ & $69.80 \mathrm{~W}$ & 5459 & 51 & 0 \\
\hline 511 & 0 & $27.81 \mathrm{~N}$ & $69.85 \mathrm{~W}$ & 5461 & Lost & \\
\hline 512 & 5223 & $27.73 \mathrm{~N}$ & $69.82 \mathrm{~W}$ & 5455 & 8 & 0 \\
\hline 513 & 5223 & $27.76 \mathrm{~N}$ & $69.87 \mathrm{~W}$ & 5455 & 8 & 0 \\
\hline 514 & 5223 & $27.71 \mathrm{~N}$ & $69.87 \mathrm{~W}$ & 5455 & 8 & 0 \\
\hline 515 & & $27.73 \mathrm{~N}$ & $69.85 \mathrm{~W}$ & 5455 & 45 & 0 \\
\hline 516 & 0 & $27.73 \mathrm{~N}$ & $69.80 \mathrm{~W}$ & & 2 & 0 \\
\hline 517 & 172 & $39.19 \mathrm{~N}$ & $70.00 \mathrm{~W}$ & 2647 & 363 & + \\
\hline 518 & 2126 & $33.59 \mathrm{~N}$ & $62.49 \mathrm{~W}$ & 3138 & 134 & 0 \\
\hline 519 & 2073 & $33.49 \mathrm{~N}$ & $62.47 \mathrm{~W}$ & 3088 & 134 & 0 \\
\hline 520 & 2115 & $33.51 \mathrm{~N}$ & $62.61 \mathrm{~W}$ & 4366 & 133 & 0 \\
\hline 521 & 482 & 28.13 & $68.68 \mathrm{~W}$ & 5265 & 128 & 0 \\
\hline 522 & 460 & $28.01 \mathrm{~N}$ & $69.75 \mathrm{~W}$ & 5462 & 128 & 0 \\
\hline
\end{tabular}


Table XV

WHOI Moored Station Data Year - 1974

\begin{tabular}{|c|c|c|c|c|c|c|}
\hline Sta.No. & $\begin{array}{c}\text { Float } \\
\text { Depth } \\
\text { m }\end{array}$ & $\begin{array}{r}\text { Latitude } \\
\text { Degrees }\end{array}$ & $\begin{array}{c}\text { Longitude } \\
\text { Degrees }\end{array}$ & $\begin{array}{c}\text { Water } \\
\text { Depth } \\
\text { m }\end{array}$ & $\begin{array}{c}\text { Duration } \\
\text { Days }\end{array}$ & Bites \\
\hline 523 & 161 & $39.43 \mathrm{~N}$ & $69.99 \mathrm{~W}$ & 2504 & 240 & 0 \\
\hline 524 & 174 & $39.13 \mathrm{~N}$ & $70.00 \mathrm{~W}$ & 2664 & 240 & 0 \\
\hline 525 & 175 & $39.12 \mathrm{~N}$ & $70.55 \mathrm{~W}$ & 2759 & 239 & $\theta$ \\
\hline 526 & 1988 & $38.78 \mathrm{~N}$ & $70.01 \mathrm{~W}$ & 3007 & 238 & 0 \\
\hline 527. & 1959 & $39.17 \mathrm{~N}$ & $69.00 \mathrm{~W}$ & 2978 & 239 & 0 \\
\hline 528 & 2307 & $38.58 \mathrm{~N}$ & $69.15 \mathrm{~W}$ & 3326 & 240 & 0 \\
\hline 529 & 2467 & $38.36 \mathrm{~N}$ & $70.00 \mathrm{~W}$ & 3480 & 239 & 0 \\
\hline 530 & 2796 & $38.02 \mathrm{~N}$ & $69.99 \mathrm{~W}$ & 3815 & 245 & 0 \\
\hline 531 & 2908 & $38.01 \mathrm{~N}$ & $69.27 \mathrm{~W}$ & 3291 & 243 & 0 \\
\hline 532 & 3191 & $37.49 \mathrm{~N}$ & $69.32 \mathrm{~W}$ & 4210 & 244 & 0 \\
\hline 533 & 3151 & $37.51 \mathrm{~N}$ & $70.00 \mathrm{~W}$ & 4182 & 244 & 0 \\
\hline 534 & 3308 & $37.02 \mathrm{~N}$ & $69.98 \mathrm{~W}$ & 4339 & 245 & 0 \\
\hline 535 & 3411 & $36.99 \mathrm{~N}$ & $69.33 \mathrm{~W}$ & 4450 & 243 & 0 \\
\hline 536 & 3437 & $36.50 \mathrm{~N}$ & $69.33 \mathrm{~W}$ & 4468 & 243 & 0 \\
\hline 537 & 3431 & $36.50 \mathrm{~N}$ & $70.00 \mathrm{~W}$ & 4463 & 244 & 0 \\
\hline 538 & 465 & $28.05 \mathrm{~N}$ & $69.74 \mathrm{~W}$ & 5457 & 100 & + \\
\hline 539 & 0 & $28.02 \mathrm{~N}$ & $69.95 \mathrm{~W}$ & 5457 & 2 & 0 \\
\hline 540 & 483 & $28.15 \mathrm{~N}$ & $68.67 \mathrm{~W}$ & 5265 & 100 & 0 \\
\hline 541 & 316 & $38.32 \mathrm{~N}$ & $69.66 \mathrm{~W}$ & 3583 & 89 & 0 \\
\hline 542 & 467 & $28.02 \mathrm{~N}$ & $69.65 \mathrm{~W}$ & 5462 & 274 & + \\
\hline 543 & 476 & $27.96 \mathrm{~N}$ & $64.97 \mathrm{~W}$ & 5363 & 272 & + \\
\hline 544 & 493 & $28.00 \mathrm{~N}$ & $60.10 \mathrm{~W}$ & 6043 & Lost & \\
\hline 545 & 477 & $27.84 \mathrm{~N}$ & $55.57 \mathrm{~W}$ & 6015 & 284 & + \\
\hline 546 & 480 & $27.90 \mathrm{~N}$ & $54.90 \mathrm{~W}$ & 5773 & 283 & $t$ \\
\hline 547 & 478 & $28.21 \mathrm{~N}$ & $54.95 \mathrm{~W}$ & 5785 & 284 & + \\
\hline 548 & 474 & $31.03 \mathrm{~N}$ & $60.07 \mathrm{~W}$ & 5550 & 279 & + \\
\hline 549 & 476 & $33.99 \mathrm{~N}$ & $60.01 \mathrm{~W}$ & 4687 & 269 & 0 \\
\hline 550 & 1977 & $36.04 \mathrm{~N}$ & $60.04 \mathrm{~W}$ & 4894 & Lost & \\
\hline 551 & 689 & $36.04 \mathrm{~N}$ & $69.98 \mathrm{~W}$ & 4533 & 130 & $\overline{0}$ \\
\hline & & & & & & \\
\hline & & & & & & \\
\hline & & & & & & \\
\hline & & & & & & \\
\hline & & & & & & \\
\hline & & & & & & \\
\hline & & & & & & \\
\hline
\end{tabular}


Table XVI

WHOI Moored Station Data Year - 1975

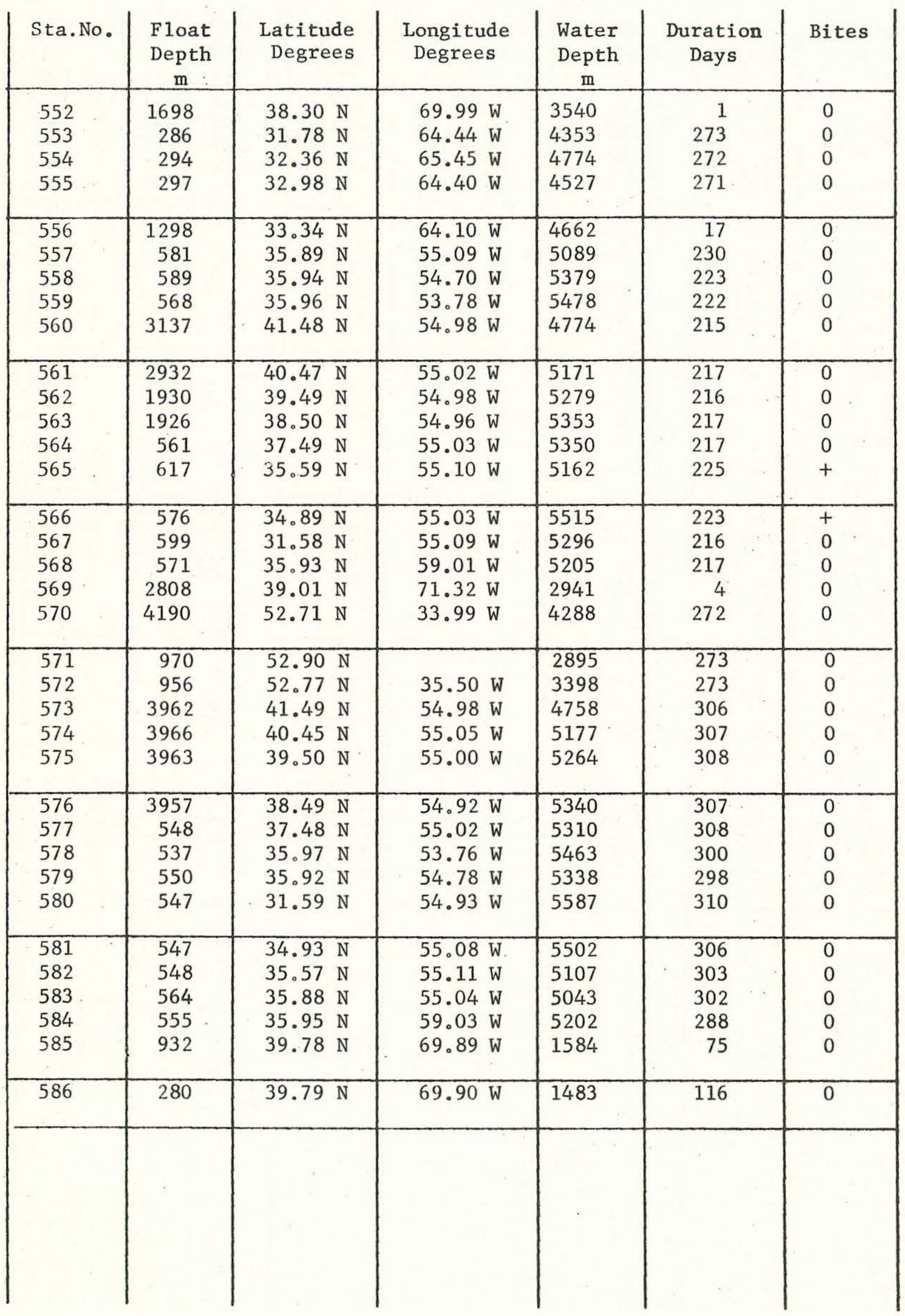


Table XVII

WHOI Moored Station Data Year -1976

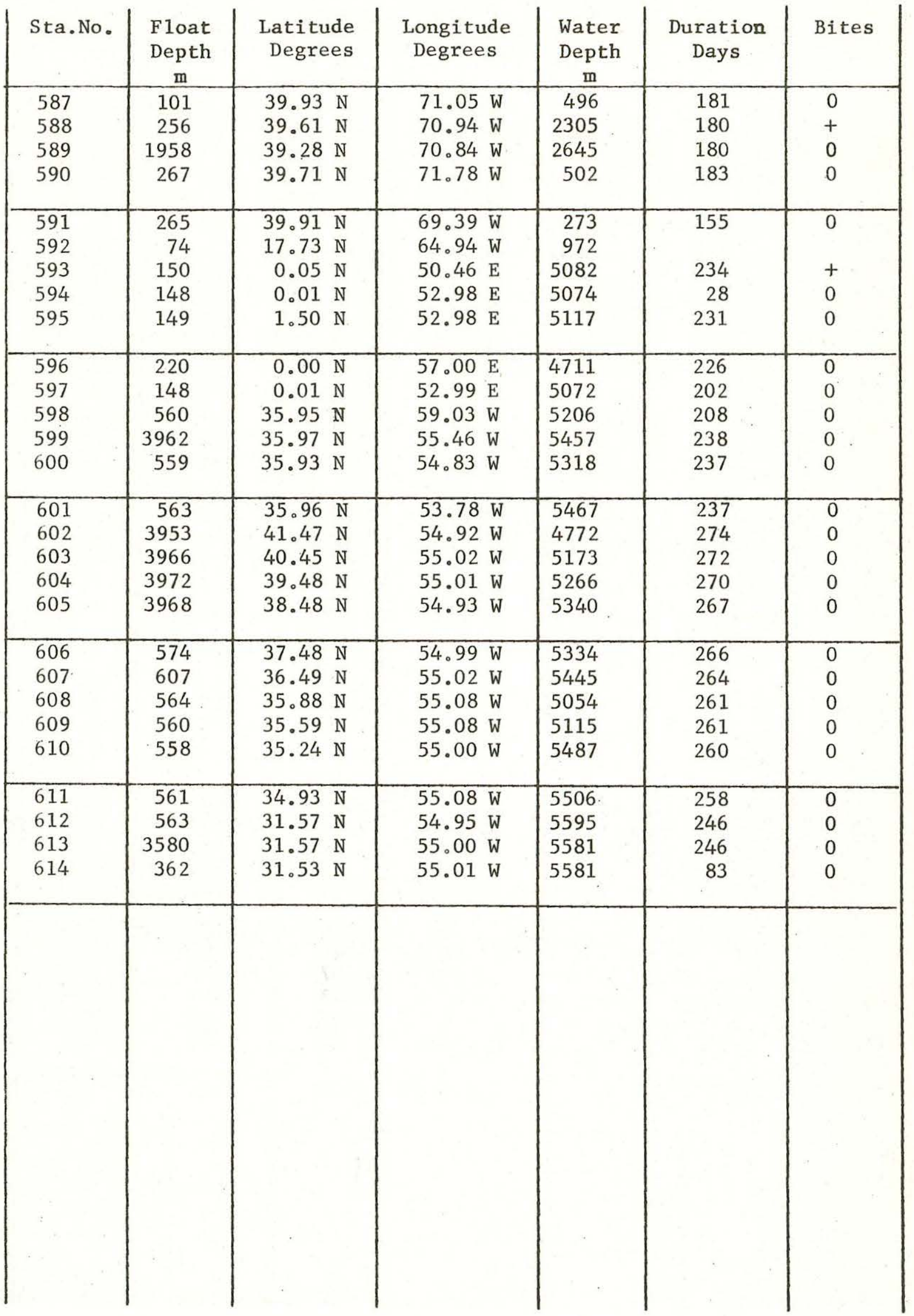


Table XVIII

WHOI Moored Station Data

Year - 1977

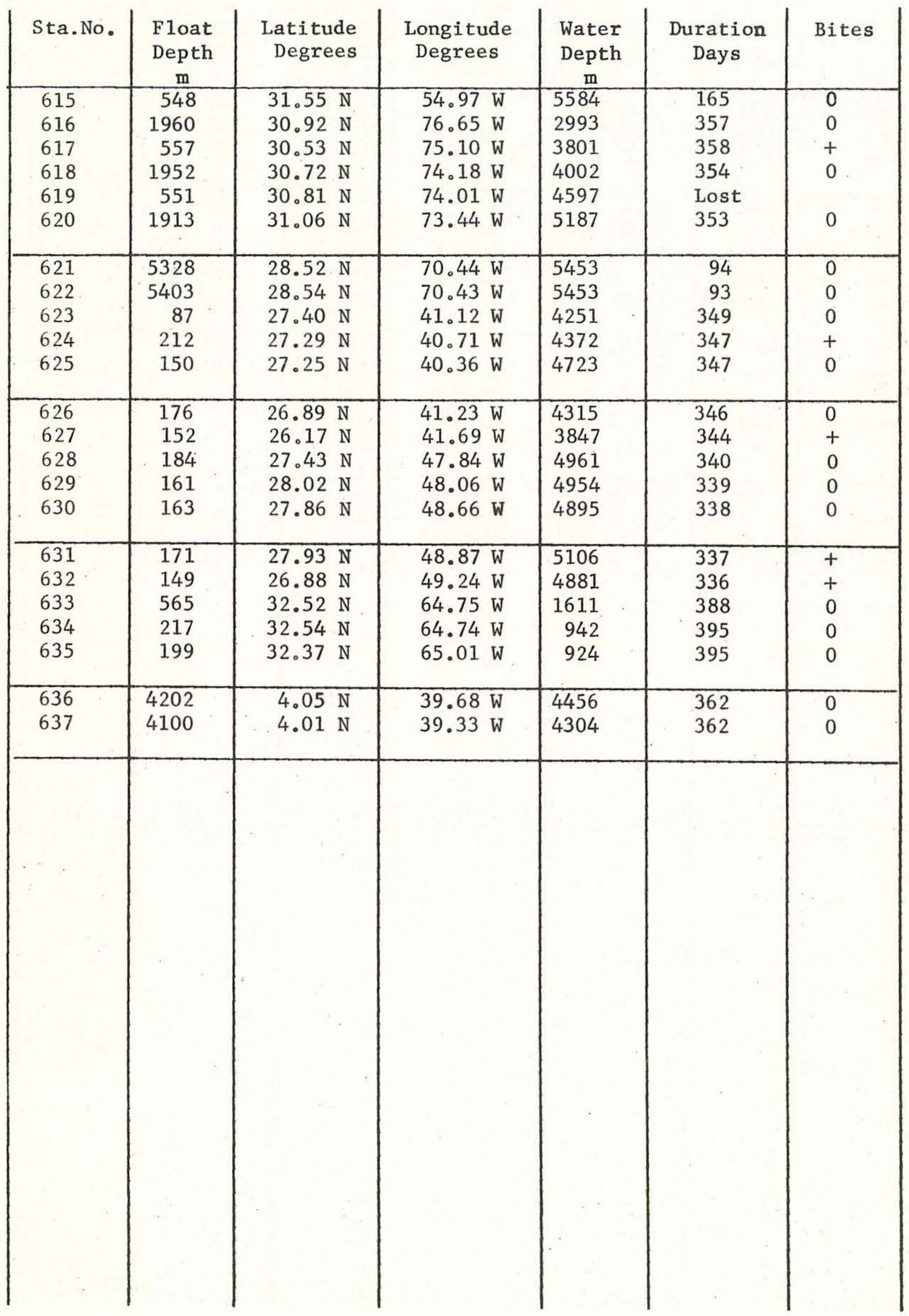


Table XIX

WHOI Moored Station Data Year - 1978

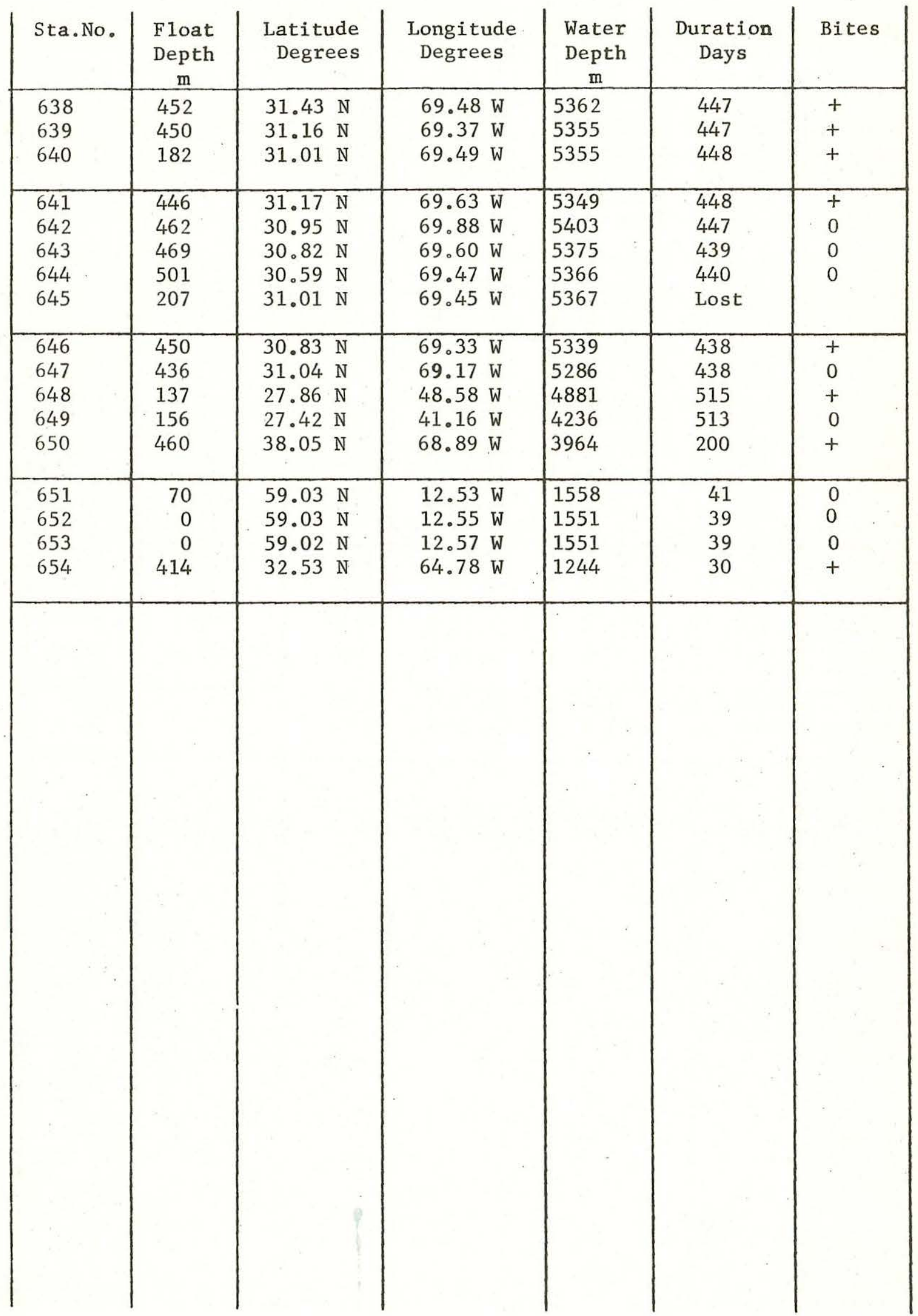


Bibliography

Prindle, Bryce and Walden, Robert G., (1976), Deep-Sea Lines Fishbite Manual", National Data Buoy Office, National Oceanic and Atmospheric Administration, Bay St. Louis, Mississippi 39520. 


\section{MANDATORY DISTRIBUTION LIST \\ FOR UNCLASSIFIED TECHNICAL REPORTS, REPRINTS, AND FINAL REPORTS PUBLISHED BY OCEANOGRAPHIC CONTRACTORS \\ OF THE OCEAN SCIENCE AND TECHNOLOGY DIVISION \\ OF THE OFFICE OF NAVAL RESEARCH}

(REVISED NOVEMBER 1978)

1 Deputy Under Secretary of Defense (Research and Advanced Technology) Military Assistant for Environmental Science Room 30129

Washington, D.C. 20301

Office of Naval Research 800 North Quincy Street

Arlington, VA 22217

ATTN: Code 483

ATTN: Code 460

ATTN: $102 B$

1 CDR J. C. Harlett, (USN)

ONR Representative Woods Hole Oceanographic Inst.

Woods Hole, MA 02543

Commanding Officer

Naval Research Laboratory

Washington, D.C. 20375

6 ATTN: Library, Code 2627
12 Defense Documentation Center Cameron Station

Alexandria, VA 22314

ATTN: DCA

Commander

Naval Oceanographic Office NSTL Station

Bay St. Louis, MS 39522

ATTN: Code 8100

ATTN: Code 6000

ATTN: Code 3300

1 NODC/NOAA

Code 0781

Wiscons in Avenue, N.W.

Washington, D.C. 20235 
UNCLASSIFIED $6 / 81$

SECURITY CLASSIFICATION OF THIS PAGE (When Date Entered)

\begin{tabular}{|c|c|}
\hline REPORT DOCUMENTATION PAGE & $\begin{array}{c}\text { READ INSTRUCTIONS } \\
\text { BEFORE COMPLETING FORM }\end{array}$ \\
\hline $\begin{array}{l}\text { 1. REPORT NUMBER } \\
\text { WHOI }-81-57\end{array}$ & 3. RECIPIENT'S CATALOG NUMBER \\
\hline $\begin{array}{l}\text { 4. TITLE (and Subtitle) } \\
\text { FACTORS CORRELATED WITH INCIDENCE OF FISHBITE }\end{array}$ & $\begin{array}{l}\text { 5. TYPE OF REPORT A PERIOD COVERED } \\
\text { Technical }\end{array}$ \\
\hline & 6. PERFORMING ORG. REPORT NUMBER \\
\hline $\begin{array}{l}\text { 7. AUTHOR(o) } \\
\text { Bryce Prindle }\end{array}$ & $\begin{array}{l}\text { 8. CONTRACT OR GRANT NUMBER(a) } \\
\text { *N00014-76-C-0197; } \\
\text { NA-79-QA-A-00475 }\end{array}$ \\
\hline $\begin{array}{l}\text { 9. PERFORMING ORGANIZATION NAME AND ADDRESS } \\
\text { Woods Hole Oceanographic Institution } \\
\text { Woods Hole, Massachusetts } 02543\end{array}$ & $\begin{array}{l}\text { 10. PROGRAMELEMENT. PROJECT, TASK } \\
\text { AREA \& WORK UNIT NUMBERS } \\
\text { * NR } 083-400\end{array}$ \\
\hline $\begin{array}{l}\text { 11. CONTROLLING OFFICE NAME AND ADDRESS } \\
\text { NORDA / National Space Technoloqy Laboratory }\end{array}$ & $\begin{array}{l}\text { 12. REPORT DATE } \\
\text { June } 1981\end{array}$ \\
\hline Bay St. Louis, MS 39529 & $\begin{array}{l}\text { 13. NUMBER OF PAGES } \\
36\end{array}$ \\
\hline 14. MONITORING AGENCY NAME \& ADDRESS(if different from Controlling Office) & $\begin{array}{l}\text { 15. SECURITY CLASS. (of this roport) } \\
\text { UnClaSSified } \\
\text { 15a. DECLASSIFICATION/DOWNGRADING } \\
\text { SCHEDULE. }\end{array}$ \\
\hline
\end{tabular}

Approved for public release; distribution unlimited.

17. DISTRIBUTION STATEMENT (of the abatract ontered in Block 20, if difforent from Roport)

18. SUPPLEMENTARY NOTES

This work was also funded by the National Data Buoy Office of the National Oceanic and Atmospheric Administration.

19. KEY WOROS (Continue on reverse alde if neceseary and Identily by block number)

1. Deep-sea Tines

2. Fishbite risk

3. Environment

20. ABSTRACT (Continue on roverse eide if neceesery and identify by block number)

Data from 399 moored stations established over the years 1967 through 1978 have been analyzed with reference to fishbite. Fishbite appears to have been a significant hazard as it was found in $22 \%$ of the lines placed within 40 degrees of the equator and where parts of the lines were at 2000 meters depth or less. Latitude, depth of water, and distance of mooring components beneath the surface were found to be correlated with incidence of fishbite; duration of a mooring was not. 


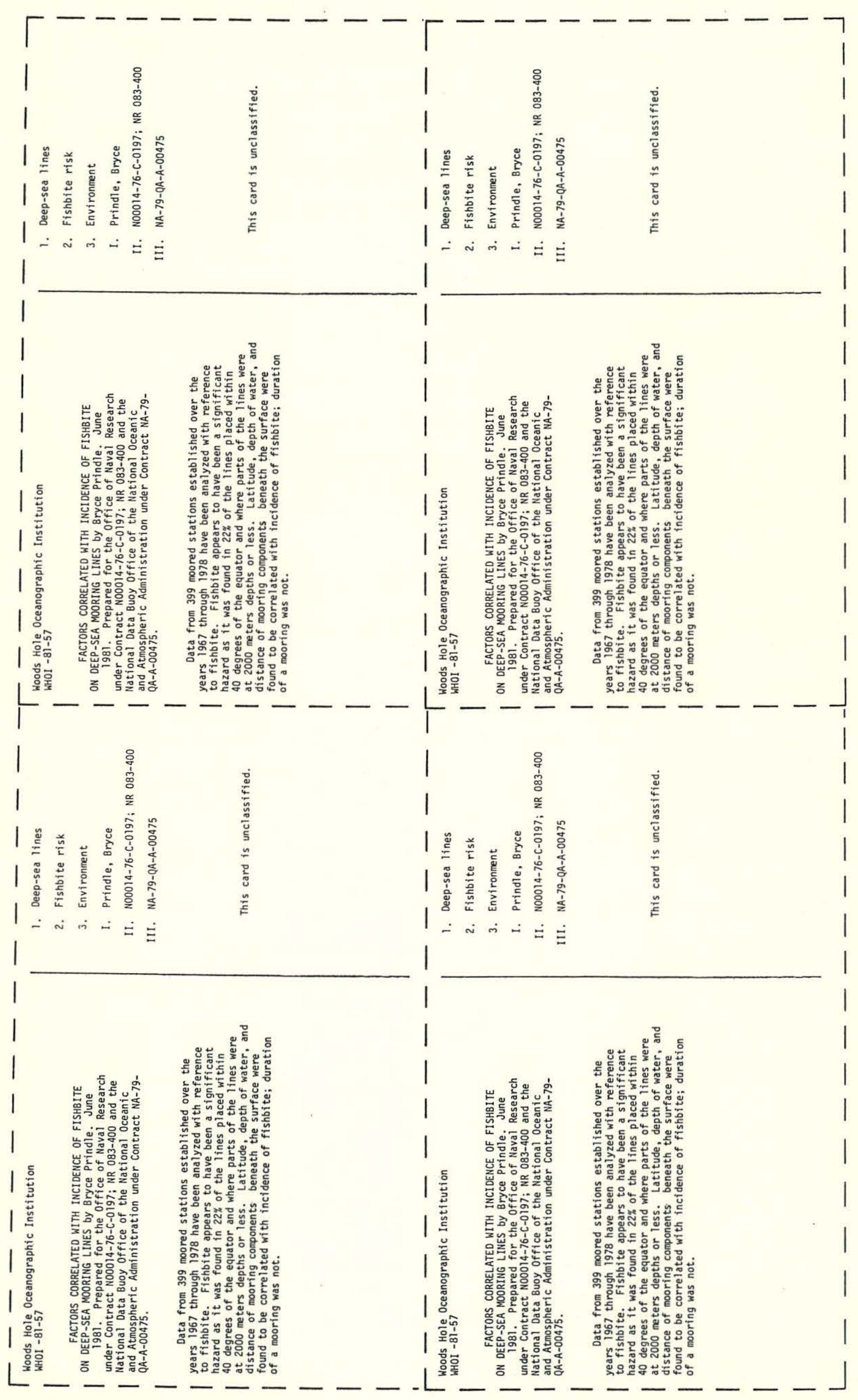

